Multiattribute Utility Theory without Expected Utility Foundations

Author(s): John M. Miyamoto and Peter Wakker

Source: Operations Research, Vol. 44, No. 2 (Mar. - Apr., 1996), pp. 313-326

Published by: INFORMS

Stable URL: http://www.jstor.org/stable/171798

Accessed: $27 /$ /04/2011 05:01

Your use of the JSTOR archive indicates your acceptance of JSTOR's Terms and Conditions of Use, available at http://www.jstor.org/page/info/about/policies/terms.jsp. JSTOR's Terms and Conditions of Use provides, in part, that unless you have obtained prior permission, you may not download an entire issue of a journal or multiple copies of articles, and you may use content in the JSTOR archive only for your personal, non-commercial use.

Please contact the publisher regarding any further use of this work. Publisher contact information may be obtained at http://www.jstor.org/action/showPublisher?publisherCode=informs.

Each copy of any part of a JSTOR transmission must contain the same copyright notice that appears on the screen or printed page of such transmission.

JSTOR is a not-for-profit service that helps scholars, researchers, and students discover, use, and build upon a wide range of content in a trusted digital archive. We use information technology and tools to increase productivity and facilitate new forms of scholarship. For more information about JSTOR, please contact support@jstor.org. 


\title{
MULTIATTRIBUTE UTILITY THEORY WITHOUT EXPECTED UTILITY FOUNDATIONS
}

\author{
JOHN M. MIYAMOTO \\ University of Washington, Seattle, Washington
}

PETER WAKKER

University of Leiden, Leiden, The Netherlands

(Received May 1993; revision received September 1994; accepted April 1995)

\begin{abstract}
Methods for determining the form of utilities are needed for the implementation of utility theory in specific decisions. An important step forward was achieved when utility theorists characterized useful parametric families of utilities and simplifying decompositions of multiattribute utilities. The standard development of these results is based on expected utility theory which is now known to be descriptively invalid. The empirical violations of expected utility impair the credibility of utility assessments. This paper shows, however, that parametric and multiattribute utility results are robust against the major violations of expected utility. They retain their validity under nonexpected utility theories that have been developed to account for actual choice behavior. To be precise, characterizations of parametric and multiattribute representations are extended to rank-dependent utility, state-dependent utility, Choquetexpected utility, and prospect theory.
\end{abstract}

$\mathrm{C}$ lassically important economic analyses made essential use of assumptions drawn from expected utility theory. Examples include results on stochastic dominance and risk aversion that were obtained by Pratt (1964), Arrow (1965), and others. The surprising discovery of Machina (1982) was that these results could be obtained in almost complete generality without requiring assumptions from expected utility theory. Another achievement of expected utility theory was the development of an operational methodology of utility analysis in which simple preference assumptions were shown to characterize tractable families of utilities, like exponential functions and additive functions of several attributes. This analytical power has been extraordinarily useful not only as a theoretical tool, but also in applied decision analyses where domain-specific utilities must be formulated.

This paper shows that, once again, results that were developed within the expected utility framework, can be extended to many forms of nonexpected utility. Specifically, characterizations of well known families of parametric utilities can be obtained in prospect theory, in the several rank-dependent theories, in the probability transformation theories of Edwards (1962) and Handa (1977), and, in effect, in all theories that deal with probabilities in a nonlinear way. Similarly, the standard multiattribute utility results can be extended to the nonexpected utility theories just mentioned. Our characterizations of utility models are based on the weakest assumptions presently available. The assumptions adopted in our analysis are compatible with either an expected or nonexpected utility basis. For expected utility, our results extend Wakker (1989, Section VII.7) to the case of arbitrary intervals. ${ }^{1} \mathrm{We}$ also extend Wakker's results to nonexpected utility models. Our main results also generalize Miyamoto's (1988) analysis of utility models, showing that the classes of utility representations axiomatized within the weak, "generic" utility theory of that paper can be axiomatized on an even weaker, and hence, more general basis. Similarly, we generalize Dyckerhoff (1994) who provided results for the decomposition of multiattribute utilities that were similar to Miyamoto (1988), and extended these to the multilinear case. For weighted utility and SSB theory, multiattribute decomposition results were given in Fishburn (1984) and characterizations of parametric families (including the linear/exponential family) were given in Fishburn (1994).

Our results have rather surprising implications for statedependent utility. When standard preference axioms for parametric and multiattribute utilities are combined with assumptions for a state-dependent utility, we find that statedependence is no longer possible. In other words, the assumptions for a state-dependent utility theory, which are strictly weaker than assumptions that imply state-independence, become equivalent in power to state-independence when combined with standard preference axioms for parametric or multiattribute utility models. Whereas the problem of identifying probabilities in a state-dependent utility theory has proven to be quite subtle (Kadane and Winkler 1988), our main results for state-dependent utility identify unique probabilities. This "solution" is unlikely to be attractive to proponents of state-dependent utility theory, however, because our assumptions eliminate state dependence. What our results show for state-dependent utility is that the development of a viable theory of utility analysis will be more difficult than has previously been recognized, 
for the standard utility analytic assumptions preclude state dependence. Our results apply to the state-dependent expected utility approach initiated by Karni (1985), and to the state- and rank-dependent approach that was initiated by Segal (1993), and extended by Green and Jullien (1988) and Chew and Wakker (1996). An elaboration of the statedependent case is given in example 1c for expected utility and in example 4 for nonexpected utility.

Finally, we note that our main results carry implications for the logical and pedagogical development of utility analysis. In the traditional approach, an initial set of axioms is adopted that imply expected utility maximization with respect to a utility of arbitrary form. Other postulates are then introduced that specify a particular form of utility. This line of development reflects the history of the subject, where expected utility foundations were laid in the 1940s and 1950s, and axioms for specific utility models were investigated in the 1960s and 1970s. Our main results show that this is not the most efficient, or conceptually economical approach. Insofar as the goal of utility analysis is to formulate assumptions that determine a specific form of utility, one can begin with weak assumptions that imply only the additive representability of utility on rank-ordered acts. Familiar postulates can then be introduced to specify a particular form of utility. State independence and identification of probabilities are obtained as useful byproducts of this analysis. This approach exploits the full power of postulates that specify particular utility forms, eliminating "foundational" assumptions that become redundant in the context of these postulates.

The organization of the paper is as follows. Section 1 states elementary definitions and assumptions concerning an additive representation for preferences over rank-ordered acts. The initial formal development is restricted to a particularly simple space of acts, namely, rank-ordered acts over a state space with only two states of nature. As we will show in Section 5, the representation theorems that we provide for rank-ordered acts over two states naturely extend to arbitrarily many states of nature. The initial restriction to two states has the further advantage of making the exposition more simple and transparent without sacrificing generality or power. Sections 2-4 develop utility representations on this elementary basis, for two states of nature. Section 2 characterizes the $\log$ /power and linear/exponential utilities for one-dimensional outcomes, Section 3 characterizes additive and multiplicative representations for two-dimensional outcomes, and Section 4 characterizes additive, multiplicative, and multilinear representations for $n$-dimensional outcomes. The usefulness of these results is demonstrated in Section 5, where classical utility results are extended to a large class of nonexpected utility theories. Appendix A shows how to replace the assumption of additive representability in Section 1 with an equivalent set of qualitative preference assumptions. Appendix B shows how monotonicity conditions, assumed in the derivations of multiattribute results (Sections 3 and 4) can be relaxed. Proofs are given in Appendix C.

\section{ELEMENTARY DEFINITIONS}

Let $\{s, t\}$ be a state space, with two distinct states (of nature). One state is the true state, the other is not true; it is not known which state is true. For example, the states might describe the result of tossing a coin, with $s$ referring to heads and $t$ to tails. We consider the general setup of decision under uncertainty, so we neither assume nor preclude that probabilities for $s$ and $t$ are given beforehand. Extensions to more than two states are given in Section 5. Let $C$ denote a set of outcomes, and let $C^{2}=C \times C$ denote the set of acts, i.e., $f=\left(f_{s}, f_{t}\right) \in C^{2}$ is the act yielding $f_{s}$ if $s$ is true and $f_{t}$ if $t$ is true. Throughout the paper, $C$ is a convex subset of $\mathrm{IR}^{\mathrm{n}}$, and more specific assumptions about $C$ will be adopted in subsequent sections.

Let $\geqslant$ denote a binary relation on $C^{2}$, whose interpretation is that of a preference relation among acts. We assume that $\geqslant$ is a weak order, i.e., it is transitive and complete (for all acts $f, g, f \geqslant g$ or $g \geqslant f$ ). As usual, we write $f>g$ if $f \geqslant g$ but not $g \geqslant f, f \sim g$ if $f \geqslant g$ and $g \geqslant$ $f, f<g$ if $g>f$, and $f \leqslant g$ if $g \geqslant f$. Preferences over riskless outcomes are represented as preferences over constant acts. For example, if $x, y \in C$, then $x \geqslant y$ is short for $(x, x) \geqslant(y, y)$. Our main results concern the set of rankordered acts, denoted $C_{\downarrow}^{2}$, and defined as the set $\left\{f \in C^{2}\right.$ : $\left.f_{s} \geqslant f_{t}\right\}$. Note that this set contains the constant acts.

In Section 5, the utility results of the paper are applied to several utility theories. In all the described applications, there exists an additive representation for $\geqslant$ on $C_{\downarrow}^{2}$, i.e., there exist functions $U_{s}: C \rightarrow$ IR and $U_{t}: C \rightarrow$ IR such that the map $f \mapsto$ $U_{s}\left(f_{s}\right)+U_{t}\left(f_{t}\right)$ preserves $\geqslant$ on $C_{\downarrow}^{2}$. Therefore this additive representation is assumed in the main body of the text; axioms for the representation are stated in Lemma 1 of Appendix A. As an example, we briefly describe the application of our results to rank-dependent utility, which is described more fully in example $3 \mathrm{a}$. On the subset of rank-ordered 50-50 lotteries $(0.5, x ; 0.5, y)$ for which $x \geqslant y$, rank-dependent utility evaluates lotteries by the function $\phi(0.5) U(x)+(1-$ $\phi(0.5)) U(y)$, for some "transformed probability" $\phi(0.5)$. The rank-ordered 50-50 lotteries are isomorphic to $C_{\downarrow}^{2}$, hence the main results of this paper can be applied, resulting in characterizations of parametric and multiattribute utility models for rank-dependent utility.

We assume that the functions $U_{s}$ and $U_{t}$ are continuous, and that both $U_{s}$ and $U_{t}$ preserve the ordering $\geqslant$ on $C$. This implies outcome monotonicity, i.e., for any $f, g \in C_{\downarrow}^{2}$, if $f_{s} \geqslant g_{s}$ and $f_{t} \geqslant g_{t}$, then $f \geqslant g$, where the consequent preference is strict if either antecedent preference is strict. The utility assumptions investigated in this paper will imply (and not presuppose) a further decomposition, namely that $U_{s}$ and $U_{t}$ are linear with respect to each other, and hence can be rewritten as $U_{s}=\pi_{s} U$ and $U_{t}=\pi_{t} U$, where $U: C \rightarrow \mathrm{IR}$. The constants $\pi_{s}$ and $\pi_{t}$ are decision weights that are positive and sum to one; the function $U$ is a utility (function). The decision weights need not be interpreted 
as probabilities; in particular cases, such as in rankdependent utility, the decision weights may have an independent interpretation within a nonexpected utility theory.

We summarize the assumptions of this paper as follows.

Structural Assumption 1. The relation $\geqslant$ is a weak order on $C^{2}$, the set of acts. There exists an additive representation $f \mapsto U_{s}\left(f_{s}\right)+U_{t}\left(f_{t}\right)$ on the rank-ordered set $C_{\downarrow}^{2}=\left\{\left(f_{s}, f_{t}\right)\right.$ $\left.\in C^{2}: f_{s} \geqslant f_{t}\right\} ;$ both $U_{s}$ and $U_{t}$ are continuous and preserve $\geqslant$ on the constant acts.

\section{PARAMETRIC FAMILIES OF UTILITIES}

In this section we consider one-dimensional outcomes and assume that larger outcomes are preferred to smaller ones, i.e., for all $x, y \in C$,

$x \geqslant y \Leftrightarrow x \geqslant y$.

This condition is called monotonicity; it implies strict increasingness of $U_{s}$ and $U_{t}$.

Our first analysis concerns the characterization of the increasing linear/exponential utilities, defined as the family of utilities $U: C \rightarrow$ IR such that one of the following holds:

$U: x \mapsto \alpha e^{\lambda x}+\tau, \quad$ for $\alpha>0, \lambda>0$, and $\tau \in \mathrm{IR}$;

$U: x \mapsto \alpha x+\tau, \quad$ for $\alpha$

$>0$ and $\tau \in \operatorname{IR}$ (parametrized by $\lambda=0)^{2}$;

$U: x \mapsto \alpha e^{\lambda x}+\tau, \quad$ for $\alpha<0, \lambda<0$, and $\tau \in$ IR .

The increasing linear/exponential family is characterized by invariance under addition of a constant: Constant absolute risk aversion on $C_{\downarrow}^{2}$ : For any $\epsilon \in \mathrm{IR}$,

$\left(f_{s}, f_{t}\right) \geqslant\left(g_{s}, g_{t}\right) \Leftrightarrow\left(f_{s}+\epsilon, f_{t}+\epsilon\right) \geqslant\left(g_{s}+\epsilon, g_{t}+\epsilon\right)$

whenever all acts in question are contained in $C_{\downarrow}^{2}$.

Theorem 1. Suppose that $C$ is a nondegenerate interval. Assume monotonicity (1), and the Structural Assumption 1. Then the following two statements are equivalent:

i. Constant absolute risk aversion holds on $C_{\downarrow}^{2}$.

ii. There exist an increasing linear/exponential utility $U: C \rightarrow$ IR and decision weights, $\pi_{s}$ and $\pi_{t}$, such that $\left(f_{s}, f_{t}\right) \mapsto$ $\pi_{s} U\left(f_{s}\right)+\pi_{t} U\left(f_{t}\right)$ represents $\geqslant$ on $C_{\downarrow}^{2}$.

Next, we turn to the characterization of the increasing $\log /$ power utilities. Let $C$ be a strictly positive interval, i.e., $C \subset \mathrm{IR}_{++}\left(\mathrm{IR}_{++}\right.$denotes the set of strictly positive real numbers). The increasing log/power functions are defined as the family of utilities $U: C \rightarrow$ IR such that one of the following holds:

$$
\begin{aligned}
& U: x \mapsto \alpha x^{\lambda}+\tau, \quad \text { for } \alpha>0, \lambda>0, \text { and } \tau \in \mathrm{IR} ; \\
& U: x \mapsto \alpha \cdot \log (x)+\tau, \quad \text { for } \alpha \\
& \quad>0 \text { and } \tau \in \mathrm{IR} \text { (parametrized by } \lambda=0 \text { ); } \\
& U: x \mapsto \alpha x^{\lambda}+\tau, \quad \text { for } \alpha<0, \lambda<0, \text { and } \tau \in \mathrm{IR} .
\end{aligned}
$$

This family of utilities is characterized by invariance under multiplication by positive real numbers: Constant relative risk aversion on $C_{\downarrow}^{2}$ : For any $\epsilon>0$,

$\left(f_{s}, f_{t}\right) \geqslant\left(g_{s}, g_{t}\right) \Leftrightarrow\left(\epsilon f_{s}, \epsilon f_{t}\right) \geqslant\left(\epsilon g_{s}, \epsilon g_{t}\right)$

whenever all acts in question are contained in $C_{\downarrow}^{2}$.

Theorem 2. Suppose that $C \subset \mathrm{IR}_{++}$is a nondegenerate interval. Assume monotonicity (1), and the Structural Assumption 1. Then the following two statements are equivalent:

i. Constant relative risk aversion holds on $C_{\downarrow}^{2}$.

ii. There exist a $\log /$ power utility $U: C \rightarrow \mathrm{IR}$ and decision weights, $\pi_{s}$ and $\pi_{t}$, such that $\left(f_{s}, f_{t}\right) \mapsto \pi_{s} U\left(f_{s}\right)+$ $\pi_{t} U\left(f_{t}\right)$ represents $\geqslant$ on $C_{\downarrow}^{2}$.

\section{MULTIATTRIBUTE UTILITY FOR TWO FACTORS}

In this section $C=C_{1} \times C_{2}$ is a product of two nondegenerate intervals, $C_{1}$ and $C_{2}{ }^{3}{ }^{3}$ That is, we deal with two product structures, first, the two-dimensional structure of $C$ that comprises all combinations of the attributes from $C_{1}$ and $C_{2}$, and, second, the two-dimensional structure $C^{2}=$ $C \times C$ that comprises the set of acts on the two-element state space $\{s, t\}$. Outcomes in $C$ are denoted as $x=\left(x_{1}\right.$, $\left.x_{2}\right)$, or $x_{1} x_{2}$ for short. Acts are denoted as $f=\left(f_{s}, f_{t}\right)=$ $\left(\left(f_{s 1}, f_{s 2}\right),\left(f_{t 1}, f_{t 2}\right)\right)$; here the outcome assigned to state $s$ is denoted as $f_{s}$, or $\left(f_{s 1}, f_{s 2}\right)$ if reference to the separate attributes is important; a similar notation is used for state $t$. When dependence on states $s$ and $t$ need not be explicitly referenced, we also denote acts as $(x, y)=\left(x_{1} x_{2}, y_{1} y_{2}\right)$. Hence, subscripts $s, t$ always refer to states and subscripts 1 and 2 always to attributes. We assume (attribute) monotonicity:

$x_{1} \geqslant y_{1}, x_{2} \geqslant y_{2}, x \neq y \Rightarrow x>y$.

The simplest class of multiattribute utilities is the additive/multiplicative family. A function $U: C \rightarrow$ IR is additive if $U: x_{1} x_{2} \mapsto U_{1}\left(x_{1}\right)+U_{2}\left(x_{2}\right)$, and multiplicative if $U: x_{1} x_{2}$ $\mapsto U_{1}\left(x_{1}\right) \cdot U_{2}\left(x_{2}\right)$, where $U_{1}: C_{1} \rightarrow$ IR and $U_{2}: C_{2} \rightarrow$ IR. A function is additive/multiplicative if it is either additive or multiplicative. The family of additive/multiplicative utilities is characterized by a utility independence property, defined as follows.

$$
C_{1} \text { is utility independent on } C_{\downarrow}^{2} \text { if }
$$

$\left(x_{1} c_{2}, y_{1} c_{2}\right) \geqslant\left(v_{1} c_{2}, w_{1} c_{2}\right)$

$\Leftrightarrow$

$\left(x_{1} d_{2}, y_{1} d_{2}\right) \geqslant\left(v_{1} d_{2}, w_{1} d_{2}\right)$

whenever all acts in question are contained in $C_{\downarrow}^{2}$. In words, if the second attribute of outcomes is fixed at a certain level, then preferences are independent of that level $\left(c_{2}\right.$ or $\left.d_{2}\right)$. Similarly, $C_{2}$ is utility independent on $C_{\downarrow}^{2}$ if preferences are independent of a fixed level of the first attribute, i.e., 


$$
\begin{aligned}
\left(c_{1} x_{2}, c_{1} y_{2}\right) & \geqslant\left(c_{1} v_{2}, c_{1} w_{2}\right) \\
& \Leftrightarrow \\
\left(d_{1} x_{2}, d_{1} y_{2}\right) & \geqslant\left(d_{1} v_{2}, d_{1} w_{2}\right)
\end{aligned}
$$

whenever all acts in question are contained in $C_{\downarrow}^{2}$. Mutual utility independence holds on $C_{\downarrow}^{2}$ if each factor is utility independent on $C_{\downarrow}^{2}$.

The definition of utility independence given here is the same as the standard definition in the multiattribute utility literature (Keeney and Raiffa 1976), except that here the condition is restricted to rank-ordered acts on a twoelement state space, $C_{\downarrow}^{2}$.

It is useful to note that the restriction of mutual utility independence to the constant acts implies that preferences between outcomes are independent of common attributes, i.e., $x_{1} c_{2} \geqslant y_{1} c_{2} \Leftrightarrow x_{1} d_{2} \geqslant y_{1} d_{2}$ and $c_{1} x_{2} \geqslant c_{1} y_{2} \Leftrightarrow d_{1} x_{2} \geqslant$ $d_{1} y_{2}$. This is the well known condition of preference independence (see Keeney and Raiffa); for two factors, the topic of this section, it is also implied by attribute monotonicity.

Theorem 3. Suppose that $C=C_{1} \times C_{2}$ is a product of nondegenerate intervals. Assume attribute monotonicity, and the Structural Assumption 1. Then the following two statements are equivalent:

i. Mutual utility independence holds on $C_{\downarrow}^{2}$.

ii. There exist an additive/multiplicative utility $U: C \rightarrow \mathrm{IR}$ and decision weights, $\pi_{s}$ and $\pi_{t}$, such that $f \mapsto \pi_{s} U\left(f_{s}\right)+$ $\pi_{t} U\left(f_{t}\right)$ represents $\geqslant$ on $C_{\downarrow}^{2}$.

Because of monotonicity, the multiplicative form in Theorem 3 does not permit changes of sign, i.e., either $U$ is strictly positive or strictly negative. The general multiplicative form in which zeroes and sign reversals can occur is more complicated on rank-ordered sets; further comments are given in Appendix B.

Theorem 3 identifies sufficient conditions for the existence of an additive or multiplicative representation, but it does not distinguish which of the two representations is valid. In the expected utility framework, it is well known that a condition called marginality is necessary and sufficient for an additive representation of attributes (Fishburn 1965)-marginality is satisfied if gambles are equal in preference whenever the gambles have identical marginal probability distributions over attributes. This condition cannot be applied in the present analysis because it assumes that the utility of a gamble is linear in probability, and that the expected utility axioms are valid. Indeed, Dyckerhoff showed for rank-dependent utility that the marginality condition implies expected utility. Miyamoto (1988) identified a condition that distinguishes additive from multiplicative representations under the less restrictive assumptions adopted here. Suppose that the assumptions of Theorem 3 are satisfied and mutual utility independence holds. Then $U$ is additive if and only if the following strengthening of utility independence holds:

$$
\begin{aligned}
& \left(x_{1} a_{2}, y_{1} b_{2}\right) \geqslant\left(v_{1} a_{2}, w_{1} b_{2}\right) \\
& \Leftrightarrow\left(x_{1} c_{2}, y_{1} d_{2}\right) \geqslant\left(v_{1} c_{2}, w_{1} d_{2}\right)
\end{aligned}
$$

whenever all acts are contained in $C_{\downarrow}^{2} . U$ is multiplicative if and only if (12) is violated for some acts in $C_{\downarrow}^{2}$.

\section{MULTIATTRIBUTE UTILITY FOR AN ARBITRARY NUMBER OF FACTORS}

Let $N=\{1, \ldots, n\}$ be the first $n$ integers, where $n \geqslant 2$, and let $C=C_{1} \times \cdots \times C_{n}$, where $C_{k}$ is a nondegenerate interval for every $k \in N$. Each outcome $x \in C$ is an $n$-tuple, $x=\left(x_{1}, \ldots, x_{n}\right)$, and the set of acts is the double product set $\left(C_{1} \times \cdots \times C_{n}\right)^{2}$. In addition, we assume $(a t$ tribute) monotonicity, i.e.,

$x_{1} \geqslant y_{1}, \ldots, x_{n} \geqslant y_{n}, x \neq y \Rightarrow x>y$.

The two-factor additive and multiplicative representations that were studied in the previous section have three natural generalizations when $n$ factors are introduced. A function $U: C \rightarrow$ IR is additive if there exist $U_{j}: C_{j} \rightarrow$ IR such that $U:\left(x_{1}, \ldots, x_{n}\right) \mapsto \sum_{j=1}^{n} U_{j}\left(x_{j}\right)$, and a function $U: C \rightarrow$ IR is multiplicative if there exist $U_{j}: C_{j} \rightarrow$ IR such that $U$ : $\left(x_{1}, \ldots, x_{n}\right) \mapsto \prod_{j=1}^{n} U_{j}\left(x_{j}\right)$. The third generalization, the multilinear case, is defined later in this section.

For $J \subset N$, and outcomes $x, y$, let $x_{J} y$ denote the outcome with attribute levels $x_{j}$ for $j \in J$ and $y_{j}$ for $j \notin J$. $C_{J}$ is utility independent on $C_{\downarrow}^{2}$ if the following holds:

$\left(x_{J} c, y_{J} c\right) \geqslant\left(v_{J} c, w_{J} c\right) \Leftrightarrow\left(x_{J} d, y_{J} d\right) \geqslant\left(v_{J} d, w_{J} d\right)$

whenever all acts in question are contained in $C_{\downarrow}^{2}$. Mutual utility independence holds on $C_{\downarrow}^{2}$ if $C_{J}$ is utility independent on $C_{\downarrow}^{2}$ for every $J \subset N$.

As in the two-factor case, the restriction of mutual utility independence to the constant acts implies that preferences between outcomes are independent of common attributes, i.e., $x_{J} c \geqslant y_{J} c \Leftrightarrow x_{J} d \geqslant y_{J} d$. This is preference independence for dimension $n \geqslant 2$.

Theorem 4. Suppose that $C=C_{1} \times \cdots \times C_{n}$, where $n \geqslant 2$ and every $C_{k}$ is a nondegenerate interval. Assume attribute monotonicity, and the Structural Assumption 1. Then the following two statements are equivalent:

i. Mutual utility independence holds on $C_{\downarrow}^{2}$.

ii. There exist an additive/multiplicative utility $U: C \rightarrow$ IR and decision weights, $\pi_{s}$ and $\pi_{t}$, such that $(x, y) \mapsto$ $\pi_{s} U(x)+\pi_{t} U(y)$ represents $\geqslant$ on $C_{\downarrow}^{2}$.

As in the two-factor case, Theorem 4 does not give the most general multiplicative form, because monotonicity excludes zeroes and sign reversals. The general multiplicative form is discussed in Appendix B. In addition, Theorem 4 only identifies sufficient conditions for an additive or multiplicative representation without indicating which representation is valid. Diagnostic conditions that distinguish the additive from the multiplicative representation have been given at the end of Section 3, and generalizations to 
$n$ factors are straightforward. We will not state these generalizations here.

Next we turn to multilinear utilities, the broadest generalization of the two-dimensional additive/multiplicative case to higher dimensions. $U: C \rightarrow$ IR is multilinear if there exist $U_{k}: C_{k} \rightarrow$ IR for each $k \in N$ and real constants, $d_{J}$, for each $J \subset N$ such that $U: x \mapsto \Sigma_{J \subset N} d_{J} \cdot \Pi_{k \in J} U_{k}\left(x_{k}\right)$.

In expected utility theory, a utility on $n$ factors is multilinear if and only if each factor is utility independent. The following theorem provides the result under weaker assumptions, thus permitting the extension to several nonexpected utility theories.

Theorem 5. Suppose that $C=C_{1} \times \cdots \times C_{n}$, where $n \geqslant 2$ and every $C_{k}$ is a nondegenerate interval. Assume attribute monotonicity, and the Structural Assumption 1. Then the following two statements are equivalent:

i. Each factor $C_{k}$ is utility independent on $C_{\downarrow}^{2}$.

ii. There exist a continuous multilinear utility $U: C \rightarrow \mathrm{IR}$ and decision weights, $\pi_{s}$ and $\pi_{t}$, such that $(x, y) \mapsto$ $\pi_{s} U(x)+\pi_{t} U(y)$ represents $\geqslant$ on $C_{\downarrow}^{2}$.

More general, nonmonotonic, multilinear forms are discussed in Appendix B.

\section{APPLICATIONS AND COMMENTS}

The preceding sections have derived standard utility results from a rather slender basis, namely, that there exists an additive representation for the preference relation on the rank-ordered acts, $C_{\downarrow}^{2}$. The results show that familiar utility representations can be characterized by the usual assumptions when the assumptions are restricted to rankordered acts. These results are robust against many of the violations of expected utility theory because violations are typically demonstrations that preference behavior is inconsistent with the global claims of expected utility theory, e.g., with the claim that decision weights are identical to stated probabilities and that strong independence assumptions like the sure-thing principle hold. Our results show that axiomatizations of standard parametric and multiattribute utility representations require only the locally additive structure within rank-ordered subsets of binary lotteries or two-outcome acts, and not the full power of expected utility theory. Hence, the techniques of this paper make possible the generalization of standard utility results to any of the so-called generalized expected utility theories that imply additive representations within rank-ordered subsets. This section gives several examples to substantiate this claim.

To bring out similarities between different utility theories, it is useful to define a common notation, terminology, and set of assumptions. We follow the standard terminology in distinguishing between decision under risk (DUR) and decision under uncertainty (DUU). In DUR, the preference relation $\geqslant$ applies to a set $P$ of probability distributions over $C$. For simplicity, we assume throughout that $P$ is the set of lotteries, i.e., probability distributions assigning a 1.0 probability to a finite subset of $C$. The notation $\left(p_{1}\right.$, $\left.x^{1} ; \ldots ; p_{m}, x^{m}\right)$ denotes a lottery yielding outcome $x^{j}$ with probability $p_{j}$ for all $j$; we use superscripts to distinguish outcomes, because subscripts already refer to attributes of outcomes. Except in example 2, we assume that outcome monotonicity holds, i.e., if $x^{k} \geqslant y^{k}$ for every $k$, then $\left(p_{1}\right.$, $\left.x^{1} ; \ldots ; p_{m}, x^{m}\right) \geqslant\left(p_{1}, y^{1} ; \ldots ; p_{m}, y^{m}\right)$, where the consequent preference is strict if $p_{k}>0$ and $x^{k}>y^{k}$ for some $k$. For any $0<p<1$, let $C_{\downarrow}^{p}$ denote the set of lotteries of the form $(p, x ; 1-p, y)$, where $x, y \in C$ and $x \geqslant y . C_{\downarrow}^{p}$ is isomorphic to $C_{\downarrow}^{2}$ under the mapping $(p, x ; 1-p, y) \mapsto$ $(x, y)$. In all of the DUR theories to be discussed, there exists an additive representation for $\geqslant$ on $C_{\downarrow}^{p}$, so that our main results can be applied under the appropriate regularity conditions.

In the case of DUU, the preference relation is over acts, which are functions from a state space $S$ to the outcome set $C$. Subsets of $S$ are called events. For simplicity of presentation we assume a finite state space $S=\left\{s_{1}, \ldots\right.$, $\left.s_{m}\right\}$ for $m \geqslant 2$. We assume that outcome monotonicity holds in the sense that if $f\left(s_{j}\right) \geqslant g\left(s_{j}\right)$ for all $j$, then $f \geqslant g$; furthermore, $f>g$ if at least one antecedent preference is strict. This implies that no state is null. For any proper subset $A \subset S$, let $\left(A, x ; A^{c}, y\right)$ denote the act assigning outcome $x$ to all $s \in A$, and $y$ to all $s \notin A$, and let $C_{\downarrow}^{A}$ denote the set of all such acts where $x \geqslant y$. This set $C_{\downarrow}^{A}$ is isomorphic to $C_{\downarrow}^{2}$ under the mapping $\left(A, x ; A^{c}, y\right) \mapsto$ $(x, y)$.

In all of the examples, we will indicate how a given utility theory implies an additive representation on a substructure of the form $C_{\downarrow}^{p}$ or $C_{\downarrow}^{A}$. Our main results can be applied to such an additive representation under appropriate regularity conditions. In each example, the characterizing preference conditions, when imposed on all acts, obviously imply the preference conditions when restricted to substructures $C_{\downarrow}^{p}$ or $C_{\downarrow}^{A}$, thus imply the restrictions for the utilities as obtained before. Note that it actually suffices, for application of the utility implications derived before, that the preference conditions have been established only on one two-dimensional substructure $C_{\downarrow}^{p}$ or $C_{\downarrow}^{A}$. Conversely, if the utilities are as described in the theorems above, then it is easy to show (and will not be elaborated here) that the preference conditions do not only hold on all substructures $C_{\downarrow}^{p}$ or $C_{\downarrow}^{A}$, but are extended naturally to the entire domain. In that manner, the following examples show how standard utility models can be characterized within the respective utility theories. As an illustration, we make the implied characterization explicit in example 3a, where rank-dependent utility is considered for the context of risk.

\section{Example 1. Additive Models}

Example 1a. Expected utility for DUR (von Neumann and Morgenstern 1944). According to this theory, there exists a utility $U: C \rightarrow$ IR that represents preferences over probability distributions through the expected utility formula. 
Take any fixed $0<p<1$. Then the function $(p, x ; 1-p$, $y) \mapsto p U(x)+(1-p) U(y)$ is an additive representation on $C_{\downarrow}^{p}$ to which our utility characterizations apply.

Example 1b. Subjective expected utility for DUU (Savage 1954). According to this theory, there exists a utility $U$ : $C \rightarrow \mathrm{IR}$, and a probability measure $P$ on $S$ that represent preferences over acts through the expected utility formula. Take any fixed event $A \subset S$ such that $0<P(A)<1$. The function $\left(A, x ; A^{c}, y\right) \mapsto P(A) U(x)+(1-P(A)) U(y)$ is an additive representation on $C_{\downarrow}^{A}$ to which our utility characterizations apply.

Examples $1 \mathrm{a}$ and $1 \mathrm{~b}$ add nothing new to the methodology of utility analysis, for the utility characterizations were proved much earlier in the expected utility and subjective expected utility framework. We mention these examples because they make clear the similarity between the utility analytic methods in these older theories, and in the theories discussed later.

Example 1c. State-dependent subjective expected utility for DUU (Karni 1985, 1992). In this theory, an act $f$ is evaluated by

$$
\sum_{i=1}^{m} P\left(s_{i}\right) U\left(f\left(s_{i}\right), s_{i}\right),
$$

where $P$ is a probability measure and $U$ is a statedependent utility function, so-called because the utility of an outcome depends on the state with which it is associated. For each state $s, U(\cdot, s)$ is assumed to be continuous. A much discussed problem with this model is that only the products $P\left(s_{i}\right) U\left(f\left(s_{i}\right), s_{i}\right)$ are inferrible from preferences, whereas the components, $P\left(s_{i}\right)$ and $U\left(f\left(s_{i}\right), s_{i}\right)$, are not separately identifiable (Drèze 1987, Karni 1985, 1992, Kadane and Winkler 1988). To see why this is the case, choose an arbitrary positive number for each state, multiply $P\left(s_{i}\right)$ by that number, divide $U\left(f\left(s_{i}\right), s_{i}\right)$ by that same number, and finally renormalize the probabilities. The result is another model that describes exactly the same preferences. Thus, probabilities and utilities are not separately identifiable. We pursue this example in some detail because our main results provide an avenue by which probabilities can be obtained.

Define $U_{j}: x \mapsto P\left(s_{j}\right) U\left(x, s_{j}\right)$ for all $j$. For convenience, we can choose an arbitrary fixed outcome, $r \in C$, and set $U_{j}(r)=0$ for all $j$. The set $C_{\downarrow}^{\left\{s_{j}\right\}}$ contains all acts $\left(\left\{s_{j}\right\}, x\right.$; $\left.\left\{s_{j}\right\}^{c}, y\right)$ with $x \geqslant y$. The function $U_{j}(x)+\sum_{i \neq j} U_{i}(y)$ is a continuous additive representation on $\left.C_{\downarrow}^{\left\{s_{j}\right.}\right\}$. Suppose that we now assume any of the parametric or multiattribute utility axioms of this paper. Then our main results establish that $\sum_{i \neq j} U_{i}(x)=\alpha U_{j}$ (plus (attribute) monotonicity $x$ ) $+\beta$ for some $\alpha>0$ and $\beta$, and every $x \in C$. Define $U^{*}: x$ $\mapsto \sum_{i=1}^{m} U_{i}(x)$. Noting that $0=\sum_{i \neq j} U_{i}(r)=\beta$, we have $U^{*}(x)=U_{j}(x)+\alpha U_{j}(x)$ for any $x \in C$. Hence, $U_{j}=\pi_{j} U^{*}$ where $\pi_{j}=1 /(1+\alpha)>0$. As this holds for every $j, 1 \leqslant j$ $\leqslant m$, the function $f \mapsto \sum_{j=1}^{m} \pi_{j} U^{*}\left(f\left(s_{j}\right)\right)$ is a stateindependent representation for the preference order over acts. Finally, the $\pi_{j}$ 's may be renormalized to sum to one. Thus, the addition of stan- dard parametric or multiattribute utility assumptions to a state-dependent subjective expected utility theory yields an expected utility model with decision weights that satisfy the conditions for probabilities. Of course, it also yields the specification of the specific utility model. Whether the decision weights derived in this analysis can be interpreted as genuine probabilities is, as always, open to debate.

Example 2. Prospect theory (Kahneman and Tversky 1979), and other DUR models in which probabilities for separate outcomes are transformed. In these theories, a continuous utility $U: C \rightarrow \mathrm{IR}$ is postulated, along with a continuous, strictly increasing probability transformation function, $\phi:[0,1] \rightarrow[0,1]$, such that $\phi(0)=0$, and $\phi(1)=$ 1. In the theories of Handa (1977) and Karmarkar (1979), a lottery $\left(p_{1}, x^{1} ; \ldots ; p_{m}, x^{m}\right)$ with distinct (nonindifferent) outcomes is evaluated by

$$
\sum_{j=1}^{m} \phi\left(p_{j}\right) U\left(x^{j}\right)
$$

For these theories, we may choose any fixed $p, 0<p<1$, and the function $(p, x ; 1-p, y) \mapsto \phi(p) U(x)+\phi(1-$ $p) U(y)$ determines an additive representation on $C_{\downarrow}^{p}$ to which our main results apply. ${ }^{4}$

In the case of the prospect theory of Kahneman and Tversky, the representation is restricted to lotteries with at most two nonzero outcomes (outcomes are real numbers here). Furthermore, different representations are postulated for lotteries that have different forms. For our results it suffices to restrict attention to the set $G_{\downarrow}^{p}$, containing all lotteries $(p, x ; 1-p, y)$ such that $x \geqslant y>0$, and the set $L_{\downarrow}^{p}$, containing all lotteries $(p, x ; 1-p, y)$ such that $0>$ $x \geqslant y$. In prospect theory, the value of such lotteries is given by

$(p, x ; 1-p, y) \mapsto \phi(p) U(x)+(1-\phi(p)) U(y)$.

On either $G_{\downarrow}^{p}$ or $L_{\downarrow}^{p}$, our main results apply to yield characterizations of parametric or multiattribute utility models within prospect theory.

We mention that Kahneman and Tversky treat the distinction between gains and losses as a psychological primitive. The sets $G_{\downarrow}^{p}$ and $L_{\downarrow}^{p}$ are sets of pure-gain and pureloss lotteries, respectively. Thus, our main results yield separate parametric or multiattribute utility axiomatizations for gains and for losses, allowing, for instance, that preferences for pure-gain lotteries $G_{\downarrow}^{p}$ satisfy constant relative risk aversion, but not preferences for pure-loss lotteries $L_{\downarrow}^{p}$, or that both gains and losses satisfy constant relative risk aversion, but with a power that is different for gains than for losses; such models were discussed in Tversky (1967). 
Example 3. Rank-dependent models. An extension of the multiattribute utility results of expected utility to rankdependent utility and Choquet-expected utility is also provided by Dyckerhoff. In particular, he also provides the result in Corollary 1c.

Example 3a. Rank-dependent utility for DUR (Quiggin 1982). Several researchers noticed that Handa's probability transformation model implied violations of stochastic dominance (Fishburn 1978, Quiggin 1982). This led Quiggin to introduce a theory, nowadays called rankdependent utility theory, that allows for transformations of probability without violating stochastic dominance. To describe the rank-dependent utility representation, first consider only lotteries of the form $\left(p_{1}, x^{1} ; \ldots ; p_{m}, x^{m}\right)$ such that $x^{1} \geqslant \cdots \geqslant x^{m}$. Rank-dependent utility theory postulates that such lotteries are evaluated by

$\sum_{j=1}^{m}\left[w\left(\sum_{k=1}^{j} p_{k}\right)-w\left(\sum_{k=1}^{j-1} p_{k}\right)\right] U\left(x^{j}\right)$,

where $U: C \rightarrow$ IR is a continuous utility, and $w:[0,1] \rightarrow$ $[0,1]$ is a continuous, strictly increasing function satisfying $w(0)=0$ and $w(1)=1$. If the outcomes are not ordered in the manner $x^{1} \geqslant \cdots \geqslant x^{m}$, then they are reordered, and a form analogous to (14) is applied.

For any fixed $p, 0<p<1$, the function $(p, x ; 1-p, y)$ $\mapsto w(p) U(x)+[1-w(p)] U(y)$ is an additive representation on $C_{\downarrow}^{p}$. Our main results apply within this structure, yielding characterizations of standard parametric and multiattribute utility models. For illustration, we make these characterizations explicit. Note that the assumption of rank-dependent utility maximization in Corollary 1 implies all conditions of Assumption 1, or their analogues, for the present context.

Corollary 1. Assume that the preference relation over lotteries is represented by rank-dependent utility.

a. Suppose that $C$ is a nondegenerate interval and $U$ is strictly increasing. Then $\left(p_{1}, x^{1} ; \ldots ; p_{m}, x^{m}\right) \geqslant\left(p_{1}, y^{1}\right.$; $\left.\ldots ; p_{m}, y^{m}\right) \Leftrightarrow\left(p_{1},\left(x^{1}+\epsilon\right) ; \ldots ; p_{m},\left(x^{m}+\epsilon\right)\right) \geqslant$ $\left(p_{1},\left(y^{1}+\epsilon\right) ; \ldots ; p_{m},\left(y^{m}+\epsilon\right)\right)$ ("constant absolute risk aversion") holds if and only if $U$ is linear/exponential.

b. Suppose that $C \subset \mathrm{IR}_{++}$is a nondegenerate interval and $U$ is strictly increasing. Then $\left(p_{1}, x^{1} ; \ldots ; p_{m}, x^{m}\right) \geqslant\left(p_{1}\right.$, $\left.y^{1}, \ldots ; p_{m}, y^{m}\right) \Leftrightarrow\left(p_{1}, \boldsymbol{\epsilon} x^{1} ; \ldots ; p_{m}, \epsilon x^{m}\right) \geqslant\left(p_{1}, \epsilon \boldsymbol{y}^{1} ;\right.$ $\ldots ; p_{m}, \epsilon y^{m}$ ) holds for any $\epsilon>0$ ("constant relative risk aversion") if and only if $U$ is a log/power utility.

c. Suppose that $C=C_{1} \times \cdots \times C_{n}$, where $n \geqslant 2$ and every $C_{k}$ is a nondegenerate interval. Assume attribute monotonicity. Then:
i. Mutual utility independence holds (whenever some at- tributes are fixed at certain levels, then preferences over lotteries over the remaining attributes are independent of the levels just described), if and only if $U$ is an additive/multiplicative utility.

ii. Each factor $C_{k}$ is utility independent (whenever all but one of the attributes are fixed at certain levels, then preferences over lotteries over the remaining attribute are independent of the levels just described), if and only if $U$ is a multilinear utility.

Example 3b. Choquet-expected utility for DUU. In this theory, introduced by Schmeidler (1989), a continuous utility, $U: C \rightarrow \mathrm{IR}$, is chosen along with a capacity, i.e., a function $W: 2^{S} \rightarrow$ IR such that $W(\varnothing)=0, W(S)=1$, and $A \supset B \Rightarrow W(A) \geqslant W(B)$. Preferences are represented by the Choquet-expected utility form: If $f\left(s_{1}\right) \geqslant \cdots \geqslant f\left(s_{m}\right)$, then $f$ is evaluated by

$$
\sum_{j=1}^{m}\left[W\left(\left\{s_{1}, \ldots, s_{j}\right\}\right)-W\left(\left\{s_{1}, \ldots, s_{j-1}\right\}\right)\right] U\left(f\left(s_{j}\right)\right) .
$$

If the states do not happen to be ordered in the manner of $f\left(s_{1}\right) \geqslant \cdots \geqslant f\left(s_{m}\right)$, they are reordered and a form analogous to (15) is applied (see Wakker 1989, Chapter VI). Let $A=\left\{s_{1}, \ldots, s_{k}\right\}$ for $1 \leqslant k<m$. The function

$$
\begin{aligned}
& \left(A, x ; A^{c}, y\right) \mapsto W\left(\left\{s_{1}, \ldots, s_{k}\right\}\right) U(x) \\
& \quad+\left[1-W\left(\left\{s_{1}, \ldots, s_{k}\right\}\right)\right] U(y)
\end{aligned}
$$

is an additive representation on $C_{\downarrow}^{A}$ to which our main results apply.

\section{Example 4. State- and Rank-Dependent Models}

Example 4a. Here we consider the generalization of rankdependent utility introduced in Segal (1989), and discussed in Wakker (1993b) and Segal (1993). A function $V: C \times$ $[0,1] \rightarrow$ IR is chosen, and a lottery $\left(p_{1}, x^{1} ; \ldots ; p_{m}, x^{m}\right)$ with $x^{1} \geqslant \cdots \geqslant x^{m}$ is evaluated by

$\sum_{j=1}^{m}\left[V\left(x^{j}, \sum_{k=1}^{j} p_{k}\right)-V\left(x^{j}, \sum_{k=1}^{j-1} p_{k}\right)\right]$.

As before, for arbitrary lotteries, the outcomes must first be rank-ordered, and then (17) applies. The function $V$ satisfies $V(x, 0) \equiv 0 \equiv V(r, p)$ for all $x, p$ with respect to a fixed reference outcome $r$. In addition, $V$ should satisfy several other natural conditions. ${ }^{5}$ For any $p, 0<p<1$, the function $(p, x ; 1-p, y) \mapsto V(x, p)+[V(y, 1)-V(y$, $p)$ ] determines an additive representation on $C_{\downarrow}^{p}$ to which our main results apply. Note also that under any of the specifications in our main results, for each fixed $0<p<1$ the functions $V(x, p)$ and $[V(x, 1)-V(x, p)]$ are related by a strictly increasing affine transformation. Hence, $V(x, 1)-V(x, p)=\alpha(p) V(x, p)+\beta(p)$ for some $\alpha(p)>$ 0 and $\beta(p) \in$ IR. Substitution of $x=r$ and $V(r, p) \equiv 0$ shows that $\beta(p) \equiv 0$, so that $V(x, p)=w(p) V(x, 1)$, for $w(p)=1 /(1+\alpha(p))$; this equation also holds for $p=0$ and $p=1$ if we define $w(0)=0$ and $w(1)=1$. Define also $U(x)=V(x, 1)$ for all outcomes $x$, so that $V(x, p)=$ $w(p) U(x)$ for all $x, p$. Now (17) reduces to

$\sum_{j=1}^{m}\left[w\left(\sum_{k=1}^{j} p_{k}\right) U\left(x^{j}\right)-w\left(\sum_{k=1}^{j-1} p_{k}\right) U\left(x^{j}\right)\right]$, 
that is, to rank-dependent utility. Here continuity of $U$ follows from continuity of $V$ in outcome, furthermore, if $V$ is continuous in probability then so is $w$, and, finally, joint monotonicity of $V$ implies that $w$ is strictly increasing. ${ }^{6}$

We find again that, as soon as this model deviates from rank-dependent utility, the classical utility characterizations are no longer possible.

Example 4b. Cumulative utility for DUU. This model was introduced by Chew and Wakker as a common generalization of all rank-dependent models. It can be interpreted as Choquet-expected utility with a state-dependent utility. A function $V: C \times 2^{S} \rightarrow$ IR is chosen. If $f\left(s_{1}\right) \geqslant \cdots \geqslant f\left(s_{m}\right)$, then act $f$ is evaluated by

$$
\begin{aligned}
\sum_{j=1}^{m}[ & V\left(f\left(s_{j}\right),\left\{s_{1}, \ldots, s_{k}\right\}\right) \\
& \left.-V\left(f\left(s_{j}\right),\left\{s_{1}, \ldots, s_{k-1}\right\}\right)\right] .
\end{aligned}
$$

If the states are not ordered in the manner of $f\left(s_{1}\right)$ $\geqslant \cdots \geqslant f\left(s_{m}\right)$, they can be reordered and a form analogous to (18) is applied (see Chew and Wakker). The function $V$ satisfies $V(x, \varnothing) \equiv 0 \equiv V(r, A)$ for all outcomes $x$ and events $A$ with respect to a fixed reference outcome $r$. In addition, $V$ should satisfy several other natural conditions. ${ }^{7}$ Let $A=\left\{s_{1}, \ldots, s_{k}\right\}$ for $1 \leqslant k<m$. Under these specifications, the function $\left(A, x ; A^{c}, y\right) \mapsto V(x, A)+[V(y$, $S)-V(y, A)]$ is an additive representation on $C_{\downarrow}^{A}$ to which our main results apply. Under the conditions studied in our main results, the present model reduces to Choquet-expected utility within each maximal comonotonic set in a manner similar to example 4a. For $m \geqslant 3$, this then reduces to a Choquet-expected utility representation on the set of all acts, as a straightforward implication of Wakker and Tversky (1993, Proposition 8.2).

Let us point out one more reason why it is possible to generalize standard results of utility analysis to theories based on rank-ordered sets of the form $C_{\downarrow}^{2}$, and thus to many models that deviate from expected utility theory. The characterizing conditions are compatible with rankordered sets in the following sense: In each characterizing condition, the acts in the antecedent preference are rankordered if and only if the acts in the consequent preference are similarly rank-ordered. This point is obvious, e.g., in the case of constant absolute risk aversion which asserts that $x \geqslant y$ if and only if $x+\epsilon \geqslant y+\epsilon$. In the case of utility independence assumptions, it was noted that mutual utility independence implies preferential independence. Thus, for example, for two dimensions we have $x_{1} c \geqslant y_{1} c$ if and only if $x_{1} d \geqslant y_{1} d$, so that a rank-ordered set can be defined on the first attribute without regard to the elements chosen from the second (or other) attributes; this is used at several places in the proofs. Axioms for standard parametric and multiattribute utility models retain their logical force when restricted to rank-ordered sets because the constraints that they impose on preferences apply to lotteries within the same rank-ordered sets.
Finally, we note that the results in this paper have been presented in the framework of DUR/DUU, with the $s_{j}$ 's referring to states of nature, but we could equally well treat the $s_{j}$ 's as referring to persons or to points of time, in which case results for welfare theory or for temporal discounting would obtain.

\section{CONCLUSION}

Our main results provide to the best of our knowledge the most general characterization of linear/exponential and $\log$ / power utility models, and the additive, multiplicative, and multilinear utility models within theories of DUR and DUU. What is new in our results is not the axioms used to characterize these utility models, but the finding that standard axioms yield the familiar utility models when added to a variety of nonexpected utility theories. Our results suggest that much of the methodology of utility analysis, as embodied in texts like Keeney and Raiffa (1976) and von Winterfeldt and Edwards (1986), can be developed upon nonexpected utility foundations.

\section{APPENDIX A. CHARACTERIZATIONS ENTIRELY IN TERMS OF PREFERENCES}

Our results have presupposed an additive representation in Structural Assumption 1; the functions $U_{s}$ and $U_{t}$ in this representation are called additive value functions. Thus, we did not obtain true characterization theorems in the sense that the conditions of these theorems were not expressed exclusively in terms of a qualitative preference relation. Here we describe how to replace the additive representation of Structural Assumption 1 with qualitative preference assumptions and thus obtain true characterization theorems. The following conditions are needed. The preference relation $\geqslant$ is continuous on $C_{\downarrow}^{2}$ if the sets $\left\{x \in C_{\downarrow}^{2}: x\right.$ $\geqslant y\}$ and $\left\{x \in C_{\downarrow}^{2}: x \leqslant y\right\}$ are closed for all $y \in C$. This condition is necessary for continuity of $U_{s}$ and $U_{t}$. The preference relation satisfies outcome monotonicity on $C_{\downarrow}^{2}$ if on that set we have

$f_{s} \geqslant g_{s}$ and $f_{t} \geqslant g_{t} \Rightarrow f \geqslant g$,

with $f>g$ if one or both antecedent preferences are strict .

This condition is necessary to guarantee that $U_{s}$ and $U_{t}$ preserve the same ordering of outcomes. Finally, the Thomsen condition is satisfied on $C_{\downarrow}^{2}$ if

$$
\begin{aligned}
& \left(f_{s}, f_{t}\right) \sim\left(g_{s}, g_{t}\right) \text { and }\left(h_{s}, g_{t}\right) \\
& \quad \sim\left(f_{s}, h_{t}\right) \Rightarrow\left(h_{s}, f_{t}\right) \sim\left(g_{s}, h_{t}\right)
\end{aligned}
$$

whenever all acts in question are contained in $C_{\downarrow}^{2}$. This condition is necessary for the additivity representation. Int will denote topological interior.

Lemma 1. Let $C$ be a convex subset of $\mathrm{IR}^{\mathrm{n}}$, and let $\geqslant$ be a weak order on $C^{2}$ that satisfies continuity, outcome monotonicity, and the Thomsen condition on $C_{\downarrow}^{2}$. Then there exist continuous functions, $U_{s}$ and $U_{t}$, from $\operatorname{Int}(C)$ to IR such that $f \mapsto U_{s}\left(f_{s}\right)+U_{t}\left(f_{t}\right)$ represents $\geqslant$ on $[\operatorname{Int}(C)]_{\downarrow}^{2}$. If $U_{s}$ 
and $U_{t}$ are linear with respect to each other, then the representation can be extended by continuity to the entire set $C_{\downarrow}^{2}$. The functions $U_{s}$ and $U_{t}$ are unique up to a positive linear transformation with common units.

Lemma 1 is proved in Wakker (1993a, Theorem 3.3(a), Proposition 3.5, and Remark 3.7), where results from additive representation theory are adapted to rank-ordered subsets of product sets. The restriction of the additive representation to the interior of $C_{\downarrow}^{2}$, or to the case of additive value functions that are linear with respect to each other, is imposed to avoid the possibility that the functions are "driven to infinity" as described in Wakker (1993a, Figure 3).

The following observation shows how characterization theorems, entirely in terms of preference conditions, could have been obtained in the main body of the text.

Observation 1. In Theorems 1-5, the Structural Assumption 1 can be dropped if the assumptions of weak ordering, continuity, the Thomsen condition on $C_{\downarrow}^{2}$, and outcome monotonicity are added to statement $\mathrm{i}$ of each theorem, and continuity of the representation is added to each statement ii.

\section{APPENDIX B. RELAXING ATTRIBUTE MONOTONICITY}

For simplicity of presentation, attribute monotonicity was assumed in the study of multiattribute utility. There is interest, however, in nonmonotonic models, for instance, if preferences on some attributes are single-peaked or if some attributes are not numerical. As another example, consider a health utility model over survival duration and health quality. People prefer longer survival to shorter survival in good health states, but the opposite preference can occur when the health state is extremely bad. Hence, preference for survival duration is neither uniformly increasing nor uniformly decreasing.

A simple way to relax attribute monotonicity is to invoke preferential independence of single attributes and an essentiality assumption. An attribute $j$ is essential on $C_{\downarrow}^{2}$ if $x_{j} c>y_{j} c$ for some $x_{j} c, y_{j} c \in C_{\downarrow}^{2}$. If attribute monotonicity is dropped from the assumptions of Theorems 3,4 , and 5, and replaced by the assumption that at least two attributes are essential, then statements $i$ of those theorems imply the corresponding statements ii; of course utility independence, which is asserted in the statements $i$, implies preferential independence of single attributes. The converse implications from statements ii to statements $\mathrm{i}$ are no longer valid because the multiplicative or multilinear forms can produce sign reversals. Sign reversals are precluded if single attributes are preferentially independent. Under preferential independence of single attributes, one can suppress the inessential attributes and rescale the essential attributes so that attribute monotonicity is satisfied. Then the results follow from those in this paper. In summary, statements $i$ and ii of Theorems 3, 4, and 5 are equivalent if attribute monotonicity is replaced by the assumption that at least two attributes are essential and $C_{j}$ is preferentially independent on $C_{\downarrow}^{2}$ for every $j$; Dyckerhoff gave this result for rank-dependent utility and Choquetexpected utility.

In general multiplicative and multilinear forms, sign reversals are possible. For expected utility, this case can be characterized by a weakening of utility independence to a "generalized utility independence," as advanced by Fishburn and Keeney $(1974,1975)$ for expected utility; see also von Stengel (1993). For our context, the generalization seems more complicated. The reason is that the generalized utility independence conditions are not compatible with rank-ordering: A crucial point for the derivation of the results in this paper was that all preference conditions that we used did not affect rank-ordering.

For the multiattribute results, there is also interest in nonnumerical attributes; for example, attributes of health may not have a quantitative description. In this case, essentiality and preferential independence conditions replace attribute monotonicity, and a richness condition is imposed to guarantee that the range of representing functions is an interval, e.g., through continuity with respect to a connected topology.

\section{APPENDIX C. PROOFS}

Proof of Theorem 1. The proof that statement $i$ of the theorem is necessary for statement ii is routine, and will be omitted. Therefore, let us assume that statement $\mathrm{i}$ holds, and prove that statement ii holds. By Lemma 1, statement ii of the theorem is proved if we can prove it for the case where $C$ is open, therefore we assume that $C$ is open. For any $\epsilon \in \mathrm{IR}^{+}$, let $C^{\epsilon}=\{x \in C: x+\epsilon \in C\}$, and let $J=$ $\left\{\epsilon \in \mathrm{IR}^{+}: C^{\epsilon}\right.$ is a nondegenerate interval $\}$. Because $C$ is a nondegenerate interval, $J$ is also a nondegenerate interval. For every $\epsilon \in J, x \mapsto U_{s}(x+\epsilon)$ and $x \mapsto U_{t}(x+\epsilon)$ are additive value functions for the same preference relation on $\left(C^{\epsilon}\right)_{\downarrow}^{2}$ as $U_{s}$ and $U_{t}$, by constant absolute risk aversion. By the uniqueness results of Lemma 1 , for each $\epsilon \in J$ there exist constants, $\sigma^{\epsilon}>0, \tau_{s}^{\epsilon}$, and $\tau_{t}^{\epsilon}$ (the $\epsilon^{\prime}$ 's are superscripts and not exponents), such that for all $x \in C^{\epsilon}$ :

$$
\begin{aligned}
& U_{s}(x+\epsilon)=\sigma^{\epsilon} U_{s}(x)+\tau_{s}^{\epsilon} \\
& U_{t}(x+\epsilon)=\sigma^{\epsilon} U_{t}(x)+\tau_{t}^{\epsilon} .
\end{aligned}
$$

Equation C.1 is a functional equation that holds if and only if $U_{s}$ is linear or exponential. This can be proved from the results in Miyamoto (1983) by substituting $x^{\prime}=$ $\exp (x), \epsilon^{\prime}=\exp (\epsilon)$, and $U_{s}^{\prime}(x)=U_{s}(\log (x))$. Under these substitutions, (C.1) becomes $U_{s}^{\prime}\left(x^{\prime} \epsilon^{\prime}\right)=\sigma^{\epsilon} U_{s}^{\prime}\left(x^{\prime}\right)+\tau_{s}^{\epsilon}$. The constants $\sigma^{\epsilon}$ and $\tau_{s}^{\epsilon}$ can be taken as constants depending on $\epsilon^{\prime}$, therefore Miyamoto's (1983) formulas (10/10), (21), and (22) apply to the equation in this form; they yield linear/exponential functions as the unique solutions to (C.1). We note in passing that Miyamoto's result generalizes similar results in Pfanzagl (1959) and Aczél (1966) to domains that are arbitrary intervals. A similar argument 
shows that linear/exponential functions are the only solutions to (C.2). Let $\lambda_{s}, \alpha_{s}$, and $\tau_{s}$ be the parameters of the linear/exponential function that solves (C.1), and let $\lambda_{t}, \alpha_{t}$, and $\tau_{t}$, be the parameters of the linear/exponential function that solves (C.2). Note that $\sigma^{\epsilon}$ appears in both (C.1) and (C.2). If $\sigma^{\epsilon} \equiv 1$, then $U_{s}$ and $U_{t}$ are linear, and hence, $0=\lambda_{s}=\lambda_{t}$. If $\sigma^{\epsilon}$ is nonconstant as a function of $\epsilon$, then $U_{s}$ and $U_{t}$ are nonlinear, and hence, $\sigma^{\epsilon}=\exp \left(\lambda_{s} \epsilon\right)=$ $\exp \left(\lambda_{t} \epsilon\right)$. Therefore $\lambda_{s}=\lambda_{t} \neq 0$ in this case. Define $U$ by $U: x \mapsto \exp (\lambda x)$ for $\lambda=\lambda_{s}=\lambda_{t}, \pi_{s}=\alpha_{s} /\left(\alpha_{s}+\alpha_{t}\right)$, and $\pi_{t}=\alpha_{t} /\left(\alpha_{s}+\alpha_{t}\right)$ in the exponential case, or $U: x \mapsto x$, $\pi_{s}=\alpha_{s} /\left(\alpha_{s}+\alpha_{t}\right)$, and $\pi_{t}=\alpha_{t} /\left(\alpha_{s}+\alpha_{t}\right)$ in the linear case. Now, $f \mapsto \pi_{s} U\left(f_{s}\right)+\pi_{t} U\left(f_{t}\right)$ represents $\geqslant$ on $C_{\downarrow}^{2}$.

Proof of Theorem 2. The theorem follows from Theorem 1 by substituting $\bar{f}_{s}=\log \left(f_{s}\right)$ and $\bar{f}_{t}=\log \left(f_{t}\right)$. We give some more comments: $\geqslant$ is defined accordingly by $\left(\bar{f}_{s}, \bar{f}_{t}\right) \geqslant\left(\bar{g}_{s}\right.$, $\left.\bar{g}_{t}\right)$ if $\left(\exp \left(\bar{f}_{s}\right), \exp \left(\bar{f}_{t}\right)\right) \geqslant\left(\exp \left(\bar{g}_{s}\right), \exp \left(\bar{g}_{t}\right)\right)$, constant relative risk aversion (9) for $\geqslant$ then reduces to $\left(\bar{f}_{s}, \bar{f}_{t}\right) \geqslant\left(\bar{g}_{s}\right.$, $\left.\bar{g}_{t}\right) \Leftrightarrow\left(\bar{f}_{s}+\bar{\epsilon}, \bar{f}_{t}+\bar{\epsilon}\right) \geqslant\left(\bar{g}_{s}+\bar{\epsilon}, \bar{g}_{t}+\bar{\epsilon}\right)$ for $\bar{\epsilon}=\log (\epsilon)$, i.e., it reduces to constant absolute risk aversion for $\triangleq$. Similarly, a linear/exponential utility model for $\risingdotseq$ reduces to a $\log /$ power utility model for $\geqslant$.

Proof of Theorem 3. For the proof that statement ii implies $i$, note that we have assumed attribute monotonicity. This implies for the multiplicative model that either $U$ is everywhere positive, or everywhere negative. Now utility independence on $C_{\downarrow}^{2}$ straightforwardly follows. Next we assume statement $\mathrm{i}$ and prove that ii holds. By Lemma 1, the result holds in general (including the form of $U$ ) if it holds in the case where $C$ is open. Therefore let us assume that $C$ is open.

Given preferential independence, the outcome preference $\left(x_{1}, c_{2}\right) \geqslant\left(y_{1}, c_{2}\right)$ does not depend on the level of $c_{2}$; hence, in the definition $\left(C_{1}\right)_{\downarrow}^{2}=\left\{\left(x_{1}, y_{1}\right) \in\left(C_{1}\right)^{2}: \exists c_{2} \in\right.$ $C_{2}:\left(\left(x_{1}, c_{2}\right) \geqslant\left(y_{1}, c_{2}\right)\right\}$, any choice of $c_{2}$ will do. Similarly, $\left(C_{2}\right)_{\downarrow} \downarrow$ is defined as the set $\left\{\left(x_{2}, y_{2}\right) \in\left(C_{2}\right)^{2}: \exists c_{1} \in C_{1}\right.$ : $\left(\left(c_{1}, x_{2}\right) \geqslant\left(c_{1}, y_{2}\right)\right\}$. We define the binary relation $\geqslant_{1}$ on $\left(C_{1}\right)_{\downarrow}^{2}$ by $\left(x_{1}, y_{1}\right) \geqslant_{1}\left(v_{1}, w_{1}\right)$ if $\left(x_{1} c_{2}, y_{1} c_{2}\right) \geqslant\left(v_{1} c_{2}, w_{1} c_{2}\right)$ for some $c_{2} \in C_{2}$. In view of utility independence, the choice of $c_{2}$ does not matter. Similarly, we define $\geqslant_{2}$ on $\left(C_{2}\right)_{\downarrow}^{2}$ by $\left(x_{2}, y_{2}\right) \geqslant_{2}\left(v_{2}, w_{2}\right)$ if and only if $\left(c_{1} x_{2}, c_{1} y_{2}\right) \geqslant$ $\left(c_{1} v_{2}, c_{1} w_{2}\right)$ for some $c_{1} \in C_{1}$.

Choose an arbitrary fixed $r_{2} \in C_{2}$, and define functions $V_{s}$ and $V_{t}$ from $C_{1}$ to IR by $V_{s}: x_{1} \mapsto U_{s}\left(x_{1} r_{2}\right)$ and $V_{t}: x_{1} \mapsto$ $U_{t}\left(x_{1} r_{2}\right)$. Utility independence on $C_{\downarrow}^{2}$ implies that for any $x_{2} \in C_{2}$, the function $\left(x_{1}, y_{1}\right) \mapsto U_{s}\left(x_{1} x_{2}\right)+U_{t}\left(y_{1} x_{2}\right)$ represents the same binary relation on $\left(C_{1}\right)_{\downarrow}^{2}$ as $\left(x_{1}, y_{1}\right) \mapsto$ $V_{s}\left(x_{1}\right)+V_{t}\left(y_{1}\right)$, i.e., they represent $\geqslant_{1}$. By the uniqueness result of Lemma $1,{ }^{8}$ there must exist real $\tau_{s}\left(x_{2}\right), \tau_{t}\left(x_{2}\right)$, and positive $\sigma\left(x_{2}\right)$ such that for all $x_{1} \in C_{1}$ :

$$
\begin{aligned}
& U_{s}\left(x_{1} x_{2}\right)=\sigma\left(x_{2}\right) V_{s}\left(x_{1}\right)+\tau_{s}\left(x_{2}\right) \\
& U_{t}\left(x_{1} x_{2}\right)=\sigma\left(x_{2}\right) V_{t}\left(x_{1}\right)+\tau_{t}\left(x_{2}\right) .
\end{aligned}
$$

Similarly, choose an arbitrary fixed $r_{1} \in C_{1}$, and define functions $W_{s}$ and $W_{t}$ from $C_{2}$ to IR by $W_{s}: x_{2} \mapsto U_{s}\left(r_{1} x_{2}\right)$ and $W_{t}: x_{2} \mapsto U_{t}\left(r_{1} x_{2}\right)$. Utility independence on $C_{\downarrow}^{2}$ implies that for all $x_{1} \in C_{1},\left(x_{2}, y_{2}\right) \mapsto U_{s}\left(x_{1} x_{2}\right)+U_{t}\left(x_{1} y_{2}\right)$ represents the same binary relation on $\left(C_{2}\right)_{\downarrow}^{2}$ as $\left(x_{2}, y_{2}\right) \mapsto$ $W_{s}\left(x_{2}\right)+W_{t}\left(y_{2}\right)$, i.e., they represent $\geqslant_{2}$. Again, by the uniqueness result of Lemma $1,{ }^{9}$ there must exist real $\tau_{s}^{\prime}\left(x_{1}\right), \tau_{t}^{\prime}\left(x_{1}\right)$, and $\sigma^{\prime}\left(x_{1}\right)$ such that

$U_{s}\left(x_{1} x_{2}\right)=\sigma^{\prime}\left(x_{1}\right) W_{s}\left(x_{2}\right)+\tau_{s}^{\prime}\left(x_{1}\right)$

$U_{t}\left(x_{1} x_{2}\right)=\sigma^{\prime}\left(x_{1}\right) W_{t}\left(x_{2}\right)+\tau_{t}^{\prime}\left(x_{1}\right)$.

Working in the expected utility framework with the entire set IR as domain, Keeney and Raiffa (Theorem 5.2) showed that (C.3) and (C.5) imply that

$$
\begin{aligned}
U_{s}\left(x_{1} x_{2}\right)= & \alpha_{s} V_{s}\left(x_{1}\right) W_{s}\left(x_{2}\right)+\beta_{s} V_{s}\left(x_{1}\right) \\
& +\gamma_{s} W_{s}\left(x_{2}\right)+\delta_{s},
\end{aligned}
$$

and (C.4) and (C.6) imply that

$$
\begin{aligned}
U_{t}\left(x_{1} x_{2}\right)= & \alpha_{t} V_{t}\left(x_{1}\right) W_{t}\left(x_{2}\right)+\beta_{t} V_{t}\left(x_{1}\right) \\
& +\gamma_{t} W_{t}\left(x_{2}\right)+\delta_{t},
\end{aligned}
$$

where $\alpha_{s}, \beta_{s}, \gamma_{s}, \delta_{s}, \alpha_{t}, \beta_{t}, \gamma_{t}$, and $\delta_{t}$ are real constants. Miyamoto (1983, Lemmas 1 and 2) showed that (C.7) and (C.8) are implied by (C.3), (C.4), (C.5), and (C.6) under the less restrictive assumptions adopted here.

Equations (C.3), (C.4), (C.7), and (C.8) imply that

$\sigma\left(x_{2}\right)=\alpha_{s} W_{s}\left(x_{2}\right)+\beta_{s}=\alpha_{t} W_{t}\left(x_{2}\right)+\beta_{t}$.

We now distinguish two cases.

Case 1. (Multiplicative Case) Suppose that $\sigma\left(x_{2}\right)$ is not constant. In this case, (C.9) implies that $\alpha_{s} \neq 0 \neq \alpha_{t}$. Algebraic manipulation of (C.7) and (C.8) yields that

$$
\begin{aligned}
& U_{s}^{*}\left(x_{1} x_{2}\right)=\sigma\left(x_{2}\right)\left[V_{s}\left(x_{1}\right)+\theta_{s}\right] \\
& U_{t}^{*}\left(x_{1} x_{2}\right)=\sigma\left(x_{2}\right)\left[V_{t}\left(x_{1}\right)+\theta_{t}\right],
\end{aligned}
$$

where $\theta_{s}=\gamma_{s} / \alpha_{s}, \theta_{t}=\gamma_{t} / \alpha_{t}, U_{s}^{*}\left(x_{1} x_{2}\right)=U_{s}\left(x_{1} x_{2}\right)+\gamma_{s} \beta_{s} / \alpha_{s}$ - $\delta_{s}$, and $U_{t}^{*}\left(x_{1} x_{2}\right)=U_{t}\left(x_{1} x_{2}\right)+\gamma_{t} \beta_{t} / \alpha_{t}-\delta_{t}$. By the uniqueness result of Lemma $1, U_{s}^{*}$ and $U_{t}^{*}$ are also additive value functions for $\geqslant$ on $C_{\downarrow}^{2}$. Moreover, by outcome monotonicity, the functions $x_{1} x_{2} \mapsto \sigma\left(x_{2}\right)\left[V_{s}\left(x_{1}\right)+\theta_{s}\right]$ and $x_{1} x_{2} \mapsto \sigma\left(x_{2}\right)\left[V_{t}\left(x_{1}\right)+\theta_{t}\right]$ represent the same preference relation $\geqslant$ on $C=C_{1} \times C_{2}$. By the standard uniqueness theorem for multiplicative representations (Krantz et al. 1971, Chapter 7), there exist $\lambda, \omega>0$ such that for all $x_{1} x_{2} \in C_{\downarrow}^{2}$,

$$
\begin{aligned}
U_{s}^{*}\left(x_{1} x_{2}\right) & =\sigma\left(x_{2}\right)\left[V_{s}\left(x_{1}\right)+\theta_{s}\right] \\
& =\lambda \sigma\left(x_{2}\right)^{\omega}\left[V_{t}\left(x_{1}\right)+\theta_{t}\right]^{\omega} \\
& =\lambda\left[U_{t}^{*}\left(x_{1} x_{2}\right)\right]^{\omega} .
\end{aligned}
$$

We know that $\sigma\left(x_{2}\right)$ is nonconstant because of attribute monotonicity, which implies that the nondegenerate $C_{2}$ is essential. Substituting two different values for $\sigma\left(x_{2}\right)$ shows, by (C.12), that $\omega=1$ and $V_{s}\left(x_{1}\right)+\theta_{s}=\lambda\left[V_{t}\left(x_{1}\right)+\theta_{t}\right]$. Hence, $U_{s}^{*}$ and $U_{t}^{*}$ are linear with respect to each other. Define $\phi: x_{1} \mapsto V_{t}\left(x_{1}\right)+\theta_{t}, U: x_{1} x_{2} \mapsto \phi\left(x_{1}\right) \sigma\left(x_{2}\right), \pi_{s}=$ $\lambda /(1+\lambda)$ and $\pi_{t}=1 /(1+\lambda)$. Then $U$ is multiplicative, 
and $\pi_{s} U$ and $\pi_{t} U$ are continuous additive value functions for $\geqslant$ on $C_{\downarrow}^{2}$.

Case 2. (Additive Case) Suppose that $\sigma\left(x_{2}\right)$ is constant. Then $\alpha_{s}=\alpha_{t}=0$ by (C.9), hence, by (C.7) and (C.8), the functions $U_{s}: x_{1} x_{2} \mapsto \beta_{s} V_{s}\left(x_{1}\right)+\gamma_{s} W_{s}\left(x_{2}\right)+\delta_{s}$ and $U_{t}: x_{1} x_{2}$ $\mapsto \beta_{t} V_{t}\left(x_{1}\right)+\gamma_{t} W_{t}\left(x_{2}\right)+\delta_{t}$ are both additive. They represent the same preference relation $\geqslant$ on $C=C_{1} \times C_{2}$; by standard uniqueness results (Krantz et al., Chapter 6), $U_{s}$ is a linear transform of $U_{t}, \beta_{s} V_{s}=\lambda \beta_{t} V_{t}+\tau_{t}$ and $\gamma_{s} W_{s}=$ $\lambda \gamma_{t} W_{t}+\zeta_{t}$ for some $\lambda>0$ and real $\tau_{t}$, $\zeta_{t}$. Define $\phi: x_{1} \mapsto$ $\beta_{t} V_{t}\left(x_{1}\right)+\tau_{t}, \psi: x_{2} \mapsto \gamma_{t} W_{t}\left(x_{2}\right)+\zeta_{t}, U: x_{1} x_{2} \mapsto \phi\left(x_{1}\right)+$ $\psi\left(x_{2}\right), \pi_{s}=\lambda /(1+\lambda)$, and $\pi_{t}=1 /(1+\lambda)$. Then $U$ is additive on $C_{1} \times C_{2}$, and $\left(x_{1} x_{2}, y_{1} y_{2}\right) \mapsto \pi_{s} U\left(x_{1} x_{2}\right)+$ $\pi_{t} U\left(y_{1} y_{2}\right)$ represents the preference order over acts.

Proof of Theorem 4. For the proof that statement ii implies $i$, note that we have assumed attribute monotonicity. This implies for the multiplicative model that either $U$ is everywhere positive or everywhere negative. Mutual utility independence on $C_{\downarrow}^{2}$ follows straightforwardly. Next let us assume $\mathrm{i}$ and prove that ii holds. Theorem 4 is equivalent to Theorem 3 when $n=2$. Therefore assume that $n>2$. To complete the proof, we must show that $U_{s}$ and $U_{t}$ are linear with respect to each other and that $U_{s}$ is either additive or multiplicative.

Continuity of $U_{s}$ and $U_{t}$ implies that $\geqslant$ is a continuous weak order, attribute monotonicity implies that every $C_{k}$ being nondegenerate- -is essential, and mutual utility independence implies preferential independence. Therefore, it follows by well known results that $\geqslant$ on $C$ is additively representable by continuous functions, $Z_{1}, \ldots, Z_{n}$ (Debreu 1960, Wakker 1989, Theorem III.6.6). In other words, $Z: x \mapsto Z_{1}\left(x_{1}\right)+\cdots+Z_{n}\left(x_{n}\right)$ represents $\geqslant$ on $C$. $Z(C)$ is a nondegenerate interval because $C$ is a product of nondegenerate intervals, and each $Z_{i}$ is continuous. Because $U_{s}$ and $U_{t}$ are continuous functions that represent the same relation, $\geqslant$, on $C$, there must exist strictly increasing continuous functions, $S$ and $T$, such that $U_{s}(x)=$ $S(Z(x))$ and $U_{t}(x)=T(Z(x))$ for every $x \in C$. Let $Z(C)_{\downarrow}^{2}$ $=\left\{(a, b) \in Z(C)^{2}: a \geqslant b\right\}$. Define an ordering $\geqslant^{\prime}$ on $Z(C)_{\downarrow}^{2}$ by the condition

$(a, b) \geqslant(c, d) \Leftrightarrow S(a)+T(b) \geqslant S(c)+T(d)$

for any $(a, b),(c, d) \in Z(C)_{\downarrow}^{2}$. It follows that

$(w, x) \geqslant(y, z) \Leftrightarrow(Z(w), Z(x)) \geqslant^{\prime}(Z(y), Z(z))$

for all acts $(w, x),(y, z) \in C_{\downarrow}^{2}$. Note that for $(w, x) \in C_{\downarrow}^{2}$, $w \geqslant x$ implies $Z(w) \geqslant Z(x)$, i.e., rank-ordering on $C^{2}$ agrees with that on $Z(C)^{2}$. Therefore, $(w, x) \mapsto(Z(w)$, $Z(x))$ is a mapping from $C_{\downarrow}^{2}$ to $Z(C)_{\downarrow}^{2}$, and $\geqslant '$ is the image relation of $\geqslant$ under that mapping.

We will show that for any $\beta \in \operatorname{Int}[Z(C)]$ there exists an open interval $B_{\beta}$ around $\beta$ on which $\geqslant^{\prime}$ satisfies constant absolute risk aversion. To prove this, we first derive the following lemma. Its proof is rather technical, and can be skipped if the reader will accept that an interval $B_{\beta}$ as described there can always be constructed; constant absolute risk aversion is derived later.

Lemma 2. For any $\beta \in \operatorname{Int}[Z(C)]$, it is possible to choose an open interval $B_{\beta} \subset Z(C)$ around $\beta$ that is sufficiently small to guarantee that for any real $\epsilon$, if $u, v, u^{\prime}, v^{\prime}, u+\epsilon, v$ $+\epsilon, u^{\prime}+\epsilon, v^{\prime}+\epsilon \in B_{\beta}$, then there exist $x_{n}, y_{n} \in C_{n}$ and $a, b, c, d \in C$ such that $Z\left(x_{n} a\right)=u, Z\left(x_{n} b\right)=v, Z\left(x_{n} c\right)=$ $u^{\prime}, Z\left(x_{n} d\right)=v^{\prime}, Z\left(y_{n} a\right)=u+\epsilon, Z\left(y_{n} b\right)=v+\epsilon, Z\left(y_{n} c\right)$ $=u^{\prime}+\epsilon$, and $Z\left(y_{n} d\right)=v^{\prime}+\epsilon$.

Proof. Choose any $\beta \in \operatorname{Int}[Z(C)]$, and let $x \in C$ satisfy $Z(x)=\beta$. Let $A_{n}=\operatorname{Int}\left\{\gamma \in\right.$ IR: $\gamma=Z\left(w_{n} x\right)$ for some $w_{n}$ $\left.\in C_{n}\right\}$ and $A_{v_{n}}=\operatorname{Int}\left\{\gamma \in\right.$ IR: $\gamma=Z\left(x_{n} w\right)$ for some $w \in$ $C$ \}; so $A_{n}$ refers to variation of $x_{n}$ in $Z(x)$, and $A_{\vee}$ refers to variation of $x_{\mathrm{v}}$ in $Z(x)$. Let $\lambda_{n}^{+}=\sup \{\alpha \in$ IR: $\alpha=\gamma-$ $\beta$ for some $\left.\gamma \in A_{n}\right\}, \lambda_{n}^{-}=\inf \{\alpha \in$ IR: $\alpha=\gamma-\beta$ for some $\left.\gamma \in A_{n}\right\}, \lambda_{v n}^{+}=\sup \{\alpha \in$ IR: $\alpha=\gamma-\beta$ for some $\gamma$ $\left.\in A_{\sqrt{ }}\right\}, \lambda_{v^{-}}=\inf \left\{\alpha \in\right.$ IR: $\alpha=\gamma-\beta$ for some $\left.\gamma \in A_{v_{n}}\right\}$. Note that $+\infty \geqslant \lambda_{n}^{+}>0>\lambda_{n}^{-} \geqslant-\infty$ and $+\infty \geqslant \lambda_{v n}^{+}>0>$ $\lambda_{\vee^{-}}^{-} \geqslant-\infty$ because $\beta \in A_{n} \cap A_{\downarrow_{n}}$ and $A_{n} \cap A_{\downarrow_{n}}$ is an open interval. Let $\mu=\min \left\{\lambda_{n}^{+},-\lambda_{n}^{-}, \lambda_{v n}^{+},-\lambda_{\sqrt{ }}^{-}\right\}$. Now define $B_{\beta}$ $=\{\alpha:|\alpha-\beta|<\mu / 2\}$. Obviously, $B_{\beta}$ is an open interval. Furthermore, $B_{\beta} \subset A_{n} \cap A_{v}$ because of how $\mu$ was defined. Finally, note that for any $u, v \in B_{\beta}$, we have $|u-v|$ $\leqslant|u-\beta|+|\beta-v|<\mu$.

To prove that $B_{\beta}$ satisfies the claim in the lemma, choose any real $\epsilon$, and suppose that $u, v, u^{\prime}, v^{\prime}, u+\epsilon, v+$ $\epsilon, u^{\prime}+\epsilon, v^{\prime}+\epsilon \in B_{\beta}$, and $u \geqslant v, u^{\prime} \geqslant v^{\prime}$; this implies $-\mu \leqslant \epsilon \leqslant \mu$. Because $B_{\beta} \subset A_{\vee}$, there exist $a, b, c, d \in C$ such that $Z\left(x_{n} a\right)=u, Z\left(x_{n} b\right)=v, Z\left(x_{n} c\right)=u^{\prime}$, and $Z\left(x_{n} d\right)=v^{\prime}$. Because $-\mu \leqslant \epsilon \leqslant \mu$, there exists $t_{n} \in C_{n}$ such that $\epsilon=Z\left(t_{n} x\right)-Z(x)=Z\left(t_{n} x\right)-\beta$. Because $Z$ is additive, $\epsilon=Z\left(t_{n} a\right)-Z\left(x_{n} a\right)=Z\left(t_{n} b\right)-Z\left(x_{n} b\right)=$ $Z\left(t_{n} c\right)-Z\left(x_{n} c\right)=Z\left(t_{n} d\right)-Z\left(x_{n} d\right)$. Substituting values and rearranging terms yields $Z\left(t_{n} a\right)=u+\epsilon, Z\left(t_{n} b\right)=v+$ $\epsilon, Z\left(t_{n} c\right)=u^{\prime}+\epsilon$, and $Z\left(t_{n} d\right)=v^{\prime}+\epsilon$. We conclude that $B_{\beta}$ is as desired.

We use Lemma 2 to show that $\geqslant$ satisfies constant absolute risk aversion on $B_{\beta}$. Choose any $u, v, u^{\prime}, v^{\prime} \in B_{\beta}$ such that $u \geqslant v, u^{\prime} \geqslant v^{\prime}$, and choose any real $\epsilon$ such that also $u+\epsilon, v+\epsilon, u^{\prime}+\epsilon, v^{\prime}+\epsilon \in B_{\beta}$. We need to show that $(u, v) \geqslant{ }^{\prime}\left(u^{\prime}, v^{\prime}\right)$ if and only if (iff) $(u+\epsilon, v+\epsilon) \geqslant$ $\left(u^{\prime}+\epsilon, v^{\prime}+\epsilon\right)$. By Lemma 2 we can choose $a, b, c, d \in$ $C$ and $x_{n}, y_{n} \in C_{n}$ such that $Z\left(x_{n} a\right)=u, Z\left(x_{n} b\right)=v$, $Z\left(x_{n} c\right)=u^{\prime}, Z\left(x_{n} d\right)=v^{\prime}, Z\left(y_{n} a\right)=u+\epsilon, Z\left(y_{n} b\right)=v+$ $\epsilon, Z\left(y_{n} c\right)=u^{\prime}+\epsilon$, and $Z\left(y_{n} d\right)=v^{\prime}+\epsilon$. Then (C.14) and utility independence imply that $(u, v) \geqslant\left(u^{\prime}, v^{\prime}\right)$ iff $\left(x_{n} a\right.$, $\left.x_{n} b\right) \geqslant\left(x_{n} c, x_{n} d\right)$ iff $\left(y_{n} a, y_{n} b\right) \geqslant\left(y_{n} c, y_{n} d\right)$ iff $(u+\epsilon, v+$ $\epsilon) \geqslant^{\prime}\left(u^{\prime}+\epsilon, v^{\prime}+\epsilon\right)$. Hence, $\geqslant^{\prime}$ satisfies constant absolute risk aversion on $B_{\beta}$.

The relation $\geqslant^{\prime}$ is a continuous weak order on $\left(B_{\beta}\right)^{2}$ and there exists, by (C.13), a continuous additive representation for $\geqslant^{\prime}$ on $\left(B_{\beta}\right)^{2} \downarrow$ with strictly increasing additive value functions. By Theorem 1, there exist decision weights $\pi_{s}(\beta)$ and $\pi_{t}(\beta)$, and a linear/exponential function, $U_{\beta}: B_{\beta} \rightarrow \mathrm{IR}$, such that $(u, v) \mapsto \pi_{s}(\beta) U_{\beta}(u)+\pi_{t}(\beta) U_{\beta}(v)$ represents 
$\geqslant^{\prime}$ on $\left(B_{\beta}\right)_{\downarrow}^{2}$. By proper choice of the scale parameter $\alpha(\beta)$ and the location parameter $\tau(\beta)$ of the exponential function (see (2)-(4); the parameters depend on $\beta$ ) we can make the function $\pi_{s}(\beta) U_{\beta}$ agree with the function $S$ in (C.13), and the function $\pi_{t}(\beta) U_{\beta}$ with the function $T-k(\beta)$, where the location constant $k(\beta)$ depends on $\beta$.

The interval $\operatorname{Int}[Z(C)]$ is covered by overlapping intervals $B_{\beta}$. The decision weights $\pi_{s}(\beta), \pi_{t}(\beta)$, the parameters of the exponential functions $U_{\beta}$, and thus the parameter $k(\beta)$, must be identical on the overlapping portions of these intervals, hence they must be the same on every $B_{\beta}$, independent of $\beta \in \operatorname{Int}[Z(C)]$. Hence, $S=\pi_{s} \cdot U^{\prime}$ and $T=\pi_{t} \cdot U^{\prime}+k$, where dependence on $\beta$ can be suppressed and $U^{\prime}$ agrees with $U_{\beta}$ on each $B_{\beta}$. Now, subtracting $k,(u, v) \mapsto \pi_{s} U^{\prime}(u)+\pi_{t} U^{\prime}(v)$ represents $\geqslant$ on $\operatorname{Int}[Z(C)]_{\downarrow}^{2} . U^{\prime}$ is continuous and strictly increasing because $S$ is.

Define $U: C \rightarrow$ IR by $U: x \mapsto U^{\prime}(Z(x))$. By (C.14), the function $(x, y) \mapsto \pi_{s} U(x)+\pi_{t} U(y)$ preserves $\geqslant$ on $Z(C)_{\downarrow}^{2}$. If $U^{\prime}$ is linear, then $U$ is linear, and $U: x \mapsto \alpha Z(x)$ $+\tau$ is additive. If $U^{\prime}$ is nonlinear, i.e., $U^{\prime}: u \mapsto \alpha e^{\lambda u}+\tau$ for $\lambda \neq 0$, then let $U_{i}: x_{i} \mapsto \exp \left(\lambda Z_{i}\left(x_{i}\right)\right)$ for each $i \in N$. Subtract $\tau$, and it follows that $U: x \mapsto \alpha U_{1}\left(x_{1}\right) \ldots U_{n}\left(x_{n}\right)$ is multiplicative.

Proof of Theorem 5. We define $C_{\vee_{k}}$ as the product $X_{j \neq k} C_{j}$, and denote its elements as $x_{v_{k}}$, etc.; $\left(x_{k} z_{k}\right)$ is an alternative notation for $x_{k} z$. First suppose that statement ii holds; we derive i. Fix any $r_{\mathrm{v}} \in C_{\mathrm{V}}$, and define $U_{k}: C_{k} \rightarrow$ IR by $U_{k}$ : $x_{k} \mapsto U\left(x_{k} r_{\mathrm{V}}\right)$. By attribute monotonicity, this function is not constant. Because $U$ is multilinear, for any $z_{\mathrm{k}} \in C_{\mathrm{k}}$, the function $x_{k} \mapsto U\left(x_{k} z_{k}\right)$ is a linear transform of $U_{k}$, i.e., it is of the form $\sigma \cdot U_{k}+\tau$; by attribute monotonicity, $\sigma$ is positive. Therefore, preferences $\left(x_{k} z_{k}, y_{k} z_{k}\right) \geqslant\left(x_{k} z_{k}\right.$, $\left.y_{k} z_{k}\right)$ on $C_{\downarrow}^{2}$ are independent of $z_{\vee k}$, i.e., attribute $k$ is utility independent on $C_{\downarrow}^{2}$.

To prove that statement $\mathrm{i}$ implies ii, assume that $\mathrm{i}$ holds. By Lemma 1, the result holds in general if it holds for the case where $C$ is open, therefore assume that $C$ is open. To complete the proof, we must show that $U_{s}$ and $U_{t}$ are linear with respect to each other, and that $U_{s}$ is multilinear. The proof is by induction on the number of factors. For $n=2, C=C_{1} \times C_{2}$. Now Theorem 5 follows from Theorem 3 because for two attributes mutual utility independence is equivalent to utility independence of single factors, and a function is multilinear if and only if it is additive/multiplicative. Hence Theorem 5 holds for $n=2$.

Now assume that Theorem 5 holds for $n \geqslant 2$ factors, and let us prove it for $n+1$ factors. Let $N=\{1, \ldots, n+$ 1\}. Define the set of rank-ordered acts over $C_{k}$ by $\left(C_{k}\right)_{\downarrow}^{2}$ $=\left\{\left(x_{k}, y_{k}\right) \in\left(C_{k}\right)^{2}\right.$ such that $x_{k} z \geqslant y_{k} z$ for some $\left.z \in C\right\}$, and define the preference order over these acts by $\left(x_{k}, y_{k}\right)$ $\geqslant_{k}\left(v_{k}, w_{k}\right)$ if and only if $\left(x_{k} z, y_{k} z\right) \geqslant\left(v_{k} z, w_{k} z\right)$ for some $z$. Because of utility independence of each factor $C_{k}$, the choice of $z$ can be arbitrary, the defined sets are indeed rank-ordered sets, and, for each $z,\left(x_{k} z, y_{k} z\right) \in C_{\downarrow}^{2}$ if and only if $\left(x_{k}, y_{k}\right) \in\left(C_{k}\right)_{\downarrow}^{2}$.
For each $k \in N$, choose an arbitrary $r \in C$ and define functions $\phi_{k}$ and $\lambda_{k}$ from $C_{k}$ to IR by $\phi_{k}: x_{k} \mapsto U_{s}\left(x_{k} r\right)$ and $\lambda_{k}: x_{k} \mapsto U_{t}\left(x_{k} r\right)$. The utility independence of $C_{k}$ implies that $\left(x_{k}, y_{k}\right) \mapsto \phi_{k}\left(x_{k}\right)+\lambda_{k}\left(y_{k}\right)$ represents $\geqslant_{k}$ on $\left(C_{k}\right)_{\downarrow}^{2}$. For any $z_{\vee_{k}} \in C_{\vee_{k}},\left(x_{k}, y_{k}\right) \mapsto U_{s}\left(x_{k} z_{\vee_{k}}\right)+U_{t}\left(y_{k} z_{k}\right)$ also represents $\geqslant_{k}$ on $\left(C_{k}\right)_{\downarrow}^{2}$ because $C_{k}$ is utility independent. By the uniqueness result of Lemma 1 , there exist real $\tau_{k}\left(z_{\vee}\right), \vartheta_{k}\left(z_{k}\right)$, and positive $\sigma_{k}\left(z_{k}\right)$ for any $z_{k} \in C_{\vee_{k}}$ such that for all $x_{k} \in C_{k}$,

$U_{s}\left(x_{k} z_{\backslash k}\right)=\sigma_{k}\left(z_{\backslash k}\right) \phi_{k}\left(x_{k}\right)+\tau_{k}\left(z_{\backslash k}\right)$

$U_{t}\left(x_{k} z_{\backslash k}\right)=\sigma_{k}\left(z_{\backslash k}\right) \lambda_{k}\left(x_{k}\right)+\vartheta_{k}\left(z_{\backslash k}\right)$.

Miyamoto (1983, Lemma 2) showed that if (C.15) and (C.16) hold for every $k \in N$, then there exist constants $d_{J}$ and $e_{J}$ such that

$$
\begin{aligned}
& U_{s}: x \mapsto \sum_{J \subset N} d_{J} \cdot \prod_{k \in J} \phi_{k}\left(x_{k}\right) \\
& U_{t}: x \mapsto \sum_{J \subset N} e_{J} \cdot \prod_{k \in J} \lambda_{k}\left(x_{k}\right)
\end{aligned}
$$

for some constants $d_{J}$ and $e_{J}$. Because $U_{s}$ and $U_{t}$ are continuous, $\phi_{k}$ and $\lambda_{k}$ are also continuous for every $k \in N$. We show that $\phi_{k}$ and $\lambda_{k}$ are linear with respect to each other for every $k \in N$.

Arbitrarily choose $k \in N$, and $r_{k} \in C_{k}$. Define an ordering, $\geqslant_{r_{k}}$, on $\left(C_{\mathrm{v}}\right)^{2}$ by $\left(x_{\mathrm{k}}, y_{\mathrm{v}}\right) \geqslant_{r_{k}}\left(u_{k}, w_{\mathrm{k}}\right)$ if $\left(r_{k} x_{\mathrm{k}}, r_{k} y_{\mathrm{k}}\right)$ $\geqslant\left(r_{k} u_{k}, r_{k} w_{v_{k}}\right)$. Let $\left(C_{\vee_{k}}\right)_{\downarrow}^{2}=\left\{\left(x_{v_{k}}, y_{v_{k}}\right) \in\left(C_{v_{k}}\right)^{2}: x_{v_{k}} \geqslant_{r_{k}}\right.$ $\left.y_{\vee_{k}}\right\}$ denote the rank-ordered acts over $C_{\mathrm{k}}$ relative to $\geqslant_{r_{k}}$. This notation refers explicitly to $r_{k} \in C_{k}$ because $\geqslant_{r_{k}}$ and $\left(C_{\vee_{k}}\right)_{\downarrow}^{2}$ may change for different choices of $r_{k}$. Because $C_{\vee}$ is a product of $n$ factors, the induction hypothesis implies that there exists a multilinear function $F_{r_{k}}: C_{\mathrm{V}} \rightarrow$ IR and decision weights $\pi_{s, r_{k}}$ and $\pi_{t, r_{k}}$ such that $\left(x_{v_{k}}, y_{v_{k}}\right) \mapsto$ $\pi_{s, r_{k}} F_{r_{k}}\left(x_{\vee_{k}}\right)+\pi_{t, r_{k}} F_{r_{k}}\left(y_{v_{k}}\right)$ represents $\geqslant_{r_{k}}$ on $\left(C_{\vee_{k}}\right)_{\downarrow}^{2}$. Let $F_{r_{k}}=\sum_{J \subset M\{k\}} b_{J} \cdot \Pi_{m \in J} \omega_{m}$ be the representation of $F_{r_{k}}$ as a multilinear function, where $\omega_{m}: C_{m} \rightarrow$ IR for $m \in$ $N \backslash\{k\}$, and $b_{J}$ is a constant for each $\left.J \subset N \backslash k\right\}$. But $\left(x_{\mathrm{v}}\right.$, $\left.y_{\vee_{k}}\right) \mapsto U_{s}\left(r_{k} x_{\vee_{k}}\right)+U_{t}\left(r_{k} y_{\vee_{k}}\right)$ represents $\geqslant_{r_{k}}$ on $\left(C_{\vee_{k}}\right)_{\downarrow}^{2}$ because $U_{s}$ and $U_{t}$ represent $\geqslant$ on $C_{\downarrow}^{2}$. By the uniqueness of additive representations, there exists an $\alpha>0$ and $\tau_{s}, \tau_{t}$ such that $\alpha U_{s}\left(r_{k} x_{\mathrm{v}}\right)+\tau_{s}=\pi_{s, r_{k}} F_{r_{k}}\left(x_{\mathrm{V}_{k}}\right)$ and $\alpha U_{t}\left(r_{k} x_{\mathrm{v}_{k}}\right)+$ $\tau_{t}=\pi_{t, r_{k}} F_{r_{k}}\left(x_{\mathrm{V}}\right)$ for every $x_{\mathrm{V}} \in C_{\mathrm{V}}$. Therefore, for any $x_{\mathrm{V}}$ $\in C_{\mathrm{V}_{k}}, \quad F_{r_{k}}\left(x_{\mathrm{v}}\right)=\left(\alpha / \pi_{s, r_{k}}\right) U_{s}\left(r_{k} x_{\mathrm{v}}\right)+\left(\tau_{s} / \pi_{s, r_{k}}\right)=$ $\left(\alpha / \pi_{t, r_{k}}\right) U_{t}\left(r_{k} x_{\mathrm{v}}\right)+\left(\tau_{t} / \pi_{t, r_{k}}\right)$. Miyamoto (1983, Lemma 3) showed that if multilinear functions are equal, then their component functions are linear with respect to each other. Thus, for every $m \in N \backslash k\}$ there exist constants $\vartheta_{m}>0$ and $\zeta_{m}$ such that

$\lambda_{m}=\vartheta_{m} \phi_{m}+\zeta_{m}$.

Moreover, since $k$ was initially chosen arbitrarily, there must exist constants $\vartheta_{k}$ and $\zeta_{k}$ such that (C.19) also holds for $m=k$. Substituting $\vartheta_{m} \phi_{m}+\zeta_{m}$ for $\lambda_{m}$ for every $m \in$ $N$ in (C.18) yields,

$U_{t}: x \mapsto \sum_{J \subset N} h_{J} \cdot \prod_{m \in J} \phi_{m}\left(x_{m}\right)$, 
where $h_{J}$ is a constant for each $J \subset N$. It is possible to write a recursive formula that determines the values of $h_{j}$ in terms of the values of $\vartheta_{m}, \zeta_{m}$, and $e_{J}$, for every $m \in N$, and every $e_{J}$ in (C.18) (see Miyamoto 1983, Lemma 3). We omit this formula because it is not needed in this proof.

By the Structural Assumption 1, there exists a strictly increasing, continuous function $M$ such that $U_{s}=M \circ U_{t}$. Combining (C.17) with (C.20) yields

$$
\sum_{J \subset N} d_{J} \cdot \prod_{k \in J} \phi_{k}\left(x_{k}\right)=M\left[\sum_{J \subset N} h_{J} \cdot \prod_{k \in J} \phi_{k}\left(x_{k}\right)\right]
$$

for every $x \in C$. This implies that $M$ is linear, as can be seen by varying only one attribute $x_{k}$, and keeping all other attributes fixed. Then $M\left(\vartheta \cdot \phi_{k}\left(x_{k}\right)\right)=\vartheta^{\prime} \cdot \phi_{k}\left(x_{k}\right)$ for constants $\vartheta, \vartheta^{\prime}$, and the first derivative of $M$ is $\vartheta^{\prime} / \vartheta$, i.e., it is constant, for the arguments in question. This can be extended to the entire domain of $M$.

Let $\alpha>0$ and $\beta$ be the real constants such that $U_{t}=$ $\alpha U_{s}+\beta$. Let $U=(1+\alpha) U_{s}, \pi_{s}=1 /(1+\alpha)$ and $\pi_{t}=$ $\alpha /(1+\alpha)$. Then $U$ is continuous and multilinear because $U_{s}$ is continuous and multilinear, and $(x, y) \mapsto \pi_{s} U(x)+$ $\pi_{t} U(y)$ represents $\geqslant$ on $C_{\downarrow}^{2}$ because $(x, y) \mapsto U_{s}(x)+$ $U_{t}(y)$ represents $\geqslant$ on $C_{\downarrow}^{2}$.

\section{NOTES}

1. Thereby yielding a positive answer to the open question stated at the end of Section VII.7 there.

2. This parametrization can be justified by convergence arguments and is convenient in the proofs that follow.

3. The results of this section and the next section can be derived for more general spaces $C_{1}$ and $C_{2}$. This is briefly discussed at the end of Appendix B.

4. A complication is that in these theories, outcomes in lotteries should be distinct (and nondifferent). Hence, preference conditions and representations are initially only given on lotteries $(p, x ; 1-p, y)$ with $x>y$. To invoke our theorems, the preference conditions and representations are first extended by continuity to the case $x \sim y$.

5. First, $V$ should satisfy a joint monotonicity condition in outcome and probability, i.e., if $q>p$ and $y>x$, then $V(y, q)-V(y, p)-V(x, q)+V(x, p)>0$, with equality if $y \sim x$. Second, $V$ should be continuous in outcome. Finally, continuity in probability can be imposed. Continuity in the sense of weak convergence implies both continuity in outcome and continuity in probability.

6. Write $V(y, q)-V(y, p)-V(x, q)+V(x, p)=[w(q)$ $-w(p)][U(y)-U(x)]$. Substitution of $p=0$ and $q=1$ shows that $U$ represents preferences over certain outcomes, this in turn implies that $w(q)>w(p)$ for $q>p$.

7. $V$ should satisfy a joint monotonicity condition in outcomes and events, i.e., if $B \supset A, B \neq A$, and $y>x$, then $V(y, B)-V(y, A)-V(x, B)+V(x, A)>0$, with equality if $y \sim x$. In addition, $V$ should be continuous in outcome.
8. Here we use the implication of utility independence that, by the definition of $\geqslant_{1},\left(C_{1}\right)_{\downarrow}^{2}$ is a rank-ordered set.

9. Note that $\left(C_{2}\right)_{\downarrow}^{2}$ is a rank-ordered set.

\section{ACKNOWLEDGMENT}

Support for the first author from the NICI Institute of the University of Nijmegen, the Medical Decision Making Unit of the University of Leiden, and the Dutch Cancer Society is gratefully acknowledged. Support for the second author from the Royal Netherlands Academy of Arts and Sciences is gratefully acknowledged.

\section{REFERENCES}

ACzÉL, J. 1966. Lectures on Functional Equations and Their Applications. Academic Press, New York.

Arrow, K. J. 1965. Aspects of the Theory of Risk-Bearing. Academic Bookstore, Helsinki, Finland. Elaborated as K. J. Arrow, Essays in the Theory of Risk-Bearing. North-Holland, Amsterdam, 1971.

Chew, S. H., AND P. P. Wakker. 1996. The Comonotonic Sure-Thing Principle. J. Risk and Uncert. 12, 5-27.

Debreu, G. 1960. Topological Methods in Cardinal Utility Theory. In Mathematical Methods in the Social Sciences, K. J. Arrow, S. Karlin, and P. Suppes (eds.). Stanford University Press, Stanford, Calif., 16-26.

Dréze, J. H. 1987. Essays on Economic Decision Under Uncertainty. Cambridge University Press, London.

DyCKerhofF, R. 1994. Decomposition of Multivariate Utility Functions in Non-Additive Expected Utility Theory. $J$. Multicrit. Dec. Anal. 3, 41-58.

EDwards, W. 1962. Subjective Probabilities Inferred From Decisions. Psy. Rev. 69, 109-135.

$\rightarrow$ Fishburn, P. C. 1965 . Independence in Utility Theory With Whole Product Sets. Opns. Res. 13, 28-45.

Fishburn, P. C. 1978. On Handa's 'New Theory of Cardinal Utility' and the Maximization of Expected Return. $J$. Polit. Econ. 86, 321-324.

$\rightarrow$ Fishburn, P. C. 1984. Multiattribute Nonlinear Utility Theory. Mgmt. Sci. 30, 1301-1310.

FishBURN, P. C. 1994. Utility of Wealth in Nonlinear Utility Theory. AT\&T Bell Laboratories, Murray Hill, N. J.

Fishburn, P. C., And R. L. Keeney. 1974. Seven Independence Concepts and Continuous Multiattribute Utility Functions. J. Math. Psy. 11, 294-327.

$\rightarrow$ Fishburn, P. C., AND R. L. KeENEY. 1975. Generalized Utility Independence and Some Implications. Opns. Res. 23, 928-940.

Green, J., AND B. Jullien. 1988. Ordinal Independence in Non-Linear Utility Theory. J. Risk and Uncert. 1, 355-387 (Erratum, 1989, 2, 119).

Handa, J. 1977. Risk, Probabilities, and a New Theory of Cardinal Utility. J. Polit. Econ. 85, 97-122.

$\rightarrow$ KadAne, J. B., AND R. L. WinkLER. 1988. Separating Probability Elicitation From Utilities. J. Am. Statist. Assoc. 83, 357-363.

Kahneman, D., And A. Tversky. 1979. Prospect Theory: An Analysis of Decision Under Risk. Econometrica 47, 263-291. 
Karmarkar, U. S. 1979. Subjectively Weighted Utility and the Allais Paradox. Organiz. Behav. and Human Perform. 24, 67-72.

KarnI, E. 1985. Decision-Making Under Uncertainty: The Case of State-Dependent Preferences. Harvard University Press, Cambridge, Massachussets.

KarnI, E. 1992. Subjective Probabilities and Utility With State-Dependent Preferences. J. Risk and Uncert. 5, 107-125.

KeEney, R. L., AND H. RaIfFA. 1976. Decisions With Multiple Objectives. John Wiley, New York.

Krantz, D. H., R. D. Luce, P. Suppes, And A. Tversky. 1971. Foundations of Measurement, Vol. I: Additive and Polynomial Representations. Academic Press, New York.

Machina, M. J. 1982. Expected Utility Analysis Without the Independence Axiom. Econometrica 50, 277-323.

Miyamoto, J. M. 1983. Measurement Foundations for Multiattribute Psychophysical Theories Based on First Order Polynomials. J. Math. Psy. 27, 152-182.

Miyamoto, J. M. 1988. Generic Utility Theory: Measurement Foundations and Applications in Multiattribute Utility Theory. J. Math. Psy. 32, 357-404.

Pfanzagl, J. 1959. A General Theory of MeasurementApplications to Utility. Naval Res. Logist. Quart. 6, 283-294.

Pratt, J. W. 1964. Risk Aversion in the Small and in the Large. Econometrica 32, 122-136.

Quiggin, J. 1982. A Theory of Anticipated Utility. J. Econ. Behav. and Organ. 3, 323-343.
SAVAGE, L. J. 1954. The Foundations of Statistics. John Wiley, New York. (Second edition 1972, Dover, New York.)

SCHMEIDLER, D. 1989. Subjective Probability and Expected Utility Without Additivity. Econometrica 57, 571-587.

SEGAL, U. 1989. Anticipated Utility: A Measure Representation Approach. Ann. Opns. Res. 19, 359-373.

SEgAL, U. 1993. The Measure Representation: A Correction. J. Risk and Uncert. 6, 99-107.

TVERsky, A. 1967. Utility Theory and Additivity Analysis of Risky Choices. J. Exper. Psy. 75, 27-36.

von NeumanN, J., AND O. Morgenstern. 1944, 1947, 1953. Theory of Games and Economic Behavior. Princeton University Press, Princeton, N.J.

VON STENGEL, B. 1993. 'Closure Properties of Independence Concepts for Continuous Utilities,' Math. O. R. 18, 346-389.

von Winterfeldt, D., AND W. Edwards. 1986. Decision Analysis and Behavioral Research. Cambridge University Press, Cambridge, England.

Wakker, P. P. 1989. Additive Representations of Preferences, $A$ New Foundation of Decision Analysis. Kluwer Academic Publishers, Dordrecht.

WAKKer, P. P. 1993a. Additive Representations on RankOrdered Sets II. The Topological Approach. J. Math. Econ. 22, 1-26.

Wakker, P. P. 1993b. Counterexamples to Segal's Measure Representation Theorem. J. Risk and Uncert. 6, 91-98.

WAKKer, P. P., AND A. TVERSKy. 1993. An Axiomatization of Cumulative Prospect Theory. J. Risk and Uncert. 7, 147-176. 